\title{
GENERALIZED NORMAL BUNDLES FOR LOCALLY-FLAT IMBEDDINGS
}

\author{
BY \\ EDWARD FADELL $\left({ }^{1}\right)$
}

1. Introduction. Let $M$ denote a compact differentiable manifold, not necessarily orientable, with fundamental class $\mu \in H_{n}\left(M ; Z_{2}\right)$. Then, there exists a unique class $V_{i} \in H^{i}\left(M ; Z_{2}\right)$ such that

$$
\left\langle S q^{i} \alpha, \mu\right\rangle=\left\langle V_{i} \smile \alpha, \mu\right\rangle
$$

for every $\alpha \in H^{n-i}\left(M ; Z_{2}\right)$, and the Stiefel-Whitney classes of $M, W_{k}, 0 \leqq k$ $\leqq n$, are given by

$$
W_{k}=\sum_{i+j=k} S q^{i} V_{j}
$$

according to $\mathrm{Wu}$ (see [13] and [9]).

Since (1) remains valid for compact manifolds which are not necessarily differentiable, Wu's formula (2) may be employed to define Stiefel-Whitney classes in the more general situation. Let $W=\sum W_{k}$ denote the so-called total Stiefel-Whitney class of $M$. Then, by a simple algebraic argument $W$ is a unit in the cohomology ring $H^{*}\left(M ; Z_{2}\right)$ thereby giving rise to a unique "dual" class $\bar{W}$ such that $W \smile \bar{W}=1$. If $M$ possesses a differential structure, then the Whitney Duality Theorem identifies $\bar{W}$ geometrically in terms of the normal bundle of any differentiable imbedding of $M$ in a Euclidean space, $R^{n+k}$. Thus, in the differentiable case, one obtains information on the dimension of $\bar{W}$, namely that $\bar{W}_{i}=0$ for $i>k$ (in fact, for $i \geqq k$ ) if $M$ is differentiably imbedded in $R^{n+k}$. In the nondifferentiable case where one defines $\bar{W}$ (purely algebraically) by the condition $W \smile \bar{W}$ $=1$, it also has been shown (see Wu [13] and Haefliger [6]), at least if $M$ is a complex, that $M \subset R^{n+k}$ implies $W_{i}=0$ for $i \geqq k$.

The objective here is to prove a Whitney Duality Theorem in the topological (nondifferentiable) situation, thus giving the dual classes a geometric interpretation in terms of an appropriate "normal bundle" associated with "locally flat" imbeddings of the topological manifold $M$ in an $(n+k)$-manifold $S$. We construct first a theory of Stiefel-Whitney classes for topological manifolds (not necessarily compact or triangulable) using essentially the tangent space of Nash [10]. We then associate to every "locally flat" imbedding $M \subset S$ a "normal fiber space" and prove the Whitney Duality Theorem in this setting. We include also a proof of Wu's

Received by the editors August 26, 1963.

( ${ }^{1}$ ) This work was supported in part by the National Science Foundation Grant NSF G-23849. 
formula (2) for $M$ compact, thus showing the equivalence of our StiefelWhitney classes with those defined by (2), in the compact case. Such an approach, namely the use of a normal fiber space, has certain advantages, in addition to avoiding triangulations and compactness. First, one can obtain certain geometric results through its use and secondly one can adapt the numerous techniques used in the differentiable case to the topological situation, with the concept of $n$-plane bundle being replaced by the concept of a generalized $n$-plane bundle (see $\$ 2$ ).

Singular homology and cohomology is employed throughout and, unless otherwise clearly stated, when the coefficient group is suppressed, the coefficient group of integers $Z$ is assumed.

\section{Preliminaries.}

Definition (2.1). An $n$-manifold $M^{n}$ is a connected separable metric space which is locally homeomorphic to $R^{n}$ (Euclidean $n$-space). Thus an $n$-manifold is an ANR (separable metric).

Definition (2.2). Let $\phi: M^{n} \rightarrow M^{n+k}$ denote an imbedding of the manifold $M^{n}$ into another manifold $M^{n+k}$. $\phi$ is said to be "locally flat" if for each $p \in \phi\left(M^{n}\right)$, there exists an open subset $U_{p}$ of $M^{n+k}$ containing $p$ and a homeomorphism $f_{p}: R^{n+k} \rightarrow U_{p}, f_{p}(0)=p$ and $f_{p}^{-1}\left(\phi\left(M^{n}\right)\right)=R^{n}$, where $R^{n} \subset R^{n+k}$ are those points whose last $k$ coordinates are 0 . By identifying $M^{n}$ with $\phi\left(M^{n}\right)$ we simply say $M^{n} \subset M^{n+k}$ is a locally flat imbedding.

Definition (2.3). Let $\mathscr{F}=(E, p, B)$ and $\mathscr{F}_{0}=\left(E_{0}, p_{0}, B\right)$ denote fiber spaces in the sense of Hurewicz [8] over the same base $B . \mathscr{F}_{0}$ is called a fiber subspace of $\mathscr{F}$ provided $E_{0} \subset E, p_{0}=p \mid E_{0}$ and $\mathscr{F}$ admits a lifting function $\lambda$ with the additional property that $\lambda$ lifts paths in $B$ into paths in $E_{0}$ if the preassigned initial point is in $E_{0}$, i.e., if $e_{0} \in E_{0}$ and $\omega \in B^{I}$, then $\lambda\left(e_{0}, \omega\right) \subset E_{0}^{I}$. $\left(\mathscr{F}, \mathscr{F}_{0}\right)=\left(E, E_{0}, p, B\right)$ will be called a fibered pair if $\left(E_{0}, p_{0}, B\right)$ is a fiber subspace of $(E, p, B)$, where $E_{0} \subset E$ and $p_{0}=p \mid E_{0}$. If $\left(E, E_{0}, p, B\right)$ is a fibered pair, the fiber is a pair $\left(F, F_{0}\right)$, where $F=p^{-1}(b)$, $F_{0}=F \cap E_{0}, b \in B$.

Suppose $\left(\mathscr{F}, \mathscr{F}_{0}\right)=\left(E, E_{0}, p, B\right),\left(\mathscr{F}^{\prime}, \mathscr{F}_{0}{ }^{\prime}\right)=\left(E^{\prime}, E_{0}^{\prime}, p^{\prime}, B\right)$ are fibered pairs over the same base $B$. A map of fibered pairs $\psi:\left(\mathscr{F}, \mathscr{F}_{0}\right) \rightarrow\left(\mathscr{F}^{\prime}, \mathscr{F}_{0}{ }^{\prime}\right)$ is a map $\psi:\left(E, E_{0}\right) \rightarrow\left(E^{\prime}, E_{0}^{\prime}\right)$ such that $p^{\prime} \psi=p$, i.e., $\psi$ is fiber preserving. If $\phi:\left(\mathscr{F}, \mathscr{F}_{0}\right) \rightarrow\left(\mathscr{F}^{\prime}, \mathscr{F}_{0}{ }^{\prime}\right)$ is another map of fibered pairs, then $\phi \sim_{f} \psi$ (read fiberwise homotopic) provided there exists a homotopy $H:\left(E, E_{0}\right)$ $\times I \rightarrow\left(E^{\prime}, E_{0}^{\prime}\right)$ such that $p^{\prime}(H(x, t))=p(x), x \in E, 0 \leqq t \leqq 1$.

Definition (2.4). $\left(\mathscr{F}, \mathscr{F}_{0}\right) \sim_{f}\left(\mathscr{F}^{\prime}, \mathscr{F}_{0}{ }^{\prime}\right)$ (read fiber homotopy equivalent) if, and only if, there exist maps of fibered pairs

$$
\phi:\left(\mathscr{F}, \mathscr{F}_{0}\right) \rightleftarrows\left(\mathscr{F}^{\prime}, \mathscr{F}_{0}{ }^{\prime}\right): \psi
$$

such that $\psi \phi \sim_{f} 1$ and $\phi \psi \sim_{f} 1$. Both $\phi$ and $\psi$ will be called fiber homotopy equivalences. 
The following theorem is useful in this connection. Its proof can be constructed by a simple extension of the proofs for the corresponding result for single fiber spaces (rather than pairs) given in [4] or [2].

Theorem (2.5). Let $\left(\mathscr{F}, \mathscr{F}_{0}\right)=\left(E, E_{0}, p, B\right),\left(\mathscr{F}^{\prime}, \mathscr{F}_{0}{ }^{\prime}\right)=\left(E^{\prime}, E_{0}^{\prime}, p^{\prime}, B\right)$ denote fibered pairs over a connected space $B$ which is dominated by a locally finite polyhedron, with respective fibers $\left(F, F_{0}\right)$ and $\left(F^{\prime}, F_{0}^{\prime}\right)$. Suppose $\phi:\left(\mathscr{F}, \mathscr{F}_{0}\right)$ $\rightarrow\left(\mathscr{F}^{\prime}, \mathscr{F}_{0}^{\prime}\right)$ is a map of fibered pairs such that the restriction

$$
\phi_{0}=\phi \mid\left(F, F_{0}\right):\left(F, F_{0}\right) \rightarrow\left(F^{\prime}, F_{0}^{\prime}\right)
$$

is a homotopy equivalence of pairs. Then, $\phi$ is a fiber homotopy equivalence. In fact, if $\psi_{0}:\left(F^{\prime}, F_{0}^{\prime}\right) \rightarrow\left(F, F_{0}\right)$ is a given homotopy inverse for $\phi_{0}$, then the fiber homotopy inverse $\psi:\left(\mathscr{F}^{\prime}, \mathscr{F}_{0}{ }^{\prime}\right) \rightarrow\left(\mathscr{F}, \mathscr{F}_{0}\right)$ for $\phi$ may be chosen as an extension of $\psi_{0}$.

Let us recall the concept of locally trivial fiber subspace. Let $\mathscr{F}$ $=(E, p, B, F), \mathscr{F}_{0}=\left(E_{0}, p_{0}, B, F_{0}\right)$ be locally trivial fiber spaces over the same base $B$, with fibers $F$ and $F_{0}$, respectively. $\mathscr{F}_{0}$ is a locally trivial fiber subspace of $\mathscr{F}$ provided $E_{0} \subset E, p_{0}=p \mid E_{0}$ and for each $x \in B$ there is an open set $U$ and a homeomorphism of pairs

$$
\phi_{U}:\left(U \times F, U \times F_{0}\right) \rightarrow\left(p^{-1}(U), p_{0}^{-1}(U)\right.
$$

such that

$$
p \phi_{U}(b, z)=b, \quad(b, z) \in U \times F .
$$

Definition (2.6). $\left(\mathscr{F}, \mathscr{F}_{0}\right)$ is called a locally trivial pair if $\mathscr{F}_{0}$ is a locally trivial fiber subspace of $\mathscr{F}$.

Proposition (2.7). (See [1].) If (F, $\mathscr{F} 0$ ) is a locally trivial pair over a paracompact base $B$, then $\left(\mathscr{F}, \mathscr{F}_{0}\right)$ is a fibered pair.

If $\left(\mathscr{F}, \mathscr{F}_{0}\right)=\left(E, E_{0}, p, B\right)$ is a fibered pair with fiber

$$
\left(F, F_{0}\right)=\left(p^{-1}\left(b_{0}\right), p_{0}^{-1}\left(b_{0}\right)\right), \quad b_{0} \in B,
$$

and if $\Omega(B)$ designates the space of loops of $B$ based at $b_{0}$, then a lifting function $\lambda$ for $\left(\mathscr{F}, \mathscr{F}_{0}\right)$ induces, in the usual manner, an action

$$
\bar{\lambda}: \Omega(B) \times H_{*}\left(F, F_{0} ; G\right) \rightarrow H_{*}\left(F, F_{0} ; G^{\prime}\right)
$$

of $\Omega(B)$ on $H_{*}\left(F, F_{0}\right)$. This action is trivial provided $\bar{\lambda}(\omega, h)=h, \omega \in \Omega(B)$, $h \in H_{*}\left(F, F_{0} ; G\right)$.

Definition (2.8). The fibered pair $\left(\mathscr{F}, \mathscr{F}_{0}\right)=\left(E, E_{0}, p, B\right)$ with fiber $\left(F, F_{0}\right)$ is said to be $G$-orientable provided the action $\Omega(B) \times H_{*}\left(F, F_{0} ; G\right)$ $\rightarrow H_{*}\left(F, F_{0} ; G\right)$ (defined above) is trivial. Orientable will mean $Z$-orientable.

Proposition (2.9). If $\left(\mathscr{F}, \mathscr{F}_{0}, p, B\right)$ is a fibered pair, with fiber $\left(F, F_{0}\right)$, 
such that the base $B$ is contractible, then $\left(\mathscr{F}, \mathscr{F}_{0}\right) \sim_{f}\left(B \times F, B \times F_{0}, \pi, B\right)$, where $\pi$ is projection of first coordinate.

Proof. The proof is just the same as the corresponding result for single fiber spaces as given in [3].

\section{Generalized bundles.}

Definition (3.1). A homology $n$-plane bundle $(n$-hpb) is a fibered pair $\left(\mathscr{F}, \mathscr{F}_{0}\right)=\left(E, E_{0}, p, B\right)$ with fiber $\left(F, F_{0}\right)$ such that

$$
\begin{array}{ll}
H_{i}\left(F, F_{0}\right)=0, & i \neq n, \\
H_{n}\left(F, F_{0}\right)=Z, & i=n .
\end{array}
$$

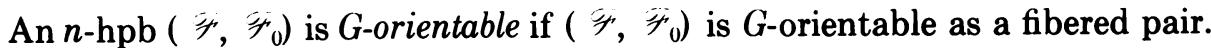

DEFinition (3.2). A generalized $n$-plane bundle $\left(n\right.$-gpb) $\left(\mathscr{F}, \mathscr{F}_{0}\right)$ $=\left(E, E_{0}, p, B\right)$ is a fibered pair with fiber $\left(F, F_{0}\right)$ and the following additional properties:

(i) There exists a cross section $\sigma: B \rightarrow E$ such that $E_{0}=E-\sigma(B)$.

(ii) $\left(F, F_{0}\right) \sim\left(R^{n}, R^{n}-0\right)$, where $R^{n}$ is a Euclidean $n$-space, $R^{n}-0$ is $R^{n}$ minus the origin and $\sim$ designates homotopy equivalence of pairs.

REMARK (3.3). Not all the conditions imposed on an $n$-gpb are necessary for the results in this paper. However, a more general concept at this point would be rather academic in this setting.

Let $D^{n} \subset R^{n}$ denote the unit $n$-ball in $R^{n}$, i.e., $D^{n}=\left\{x \in R^{n},\|x\| \leqq 1\right\}$, and let $V^{n}$ denote the interior of $D^{n}$. Furthermore, let

$$
h_{n}(x)=\frac{x}{1+\|x\|}, \quad x \in R^{n} .
$$

Then, $h_{n}$ is a homeomorphism $h_{n}: R^{n} \rightarrow V^{n}$ with inverse $g_{n}$ given by

$$
g_{n}(y)=\frac{y}{1-\|y\|}, \quad y \in V^{n} .
$$

Note that $h_{k}=h_{n} \mid R^{k}: R^{k} \rightarrow V^{k}$, for $1 \leqq k \leqq n$. A map $\gamma^{\prime}: V^{n} \times V^{n} \times D^{n} \rightarrow D^{n}$ is obtained by setting

$$
\gamma^{\prime}(y, z, w)= \begin{cases}h_{n}\left(g_{n}(w)+g_{n}(z)-g_{n}(y)\right), & w \in V^{n}, \\ w, & w \in \partial D^{n},\end{cases}
$$

$\gamma^{\prime}$ induces, in the usual fashion, a map $\gamma: V^{n} \times V^{n} \rightarrow G_{0}\left(D^{n}\right)$, where $G_{0}\left(D^{n}\right)$ is the group of homeomorphisms of $D^{n}$ (c-o topology) which are pointwise fixed on $\partial D^{n}$. We list the following standard properties of $\gamma$ :

(i) $\gamma(y, z)(y)=z, \quad(y, z) \in V^{n} \times V^{n}$;

(ii) $\gamma(y, y)=1, \quad y \in V^{n}$;

(iii) $\gamma(y, z) \mid \partial D^{n}=1, \quad(y, z) \in V^{n} \times V^{n}$.

$\gamma$ also satisfies additional properties which will be useful to us later. Let 
$D^{k}=D^{n} \cap R^{k}, V^{k}=V^{n} \cap R^{k}, 1 \leqq k \leqq n$. Furthermore, let $G_{0}^{k}\left(D^{n}\right)$ denote the subgroup of $G_{0}\left(D^{n}\right)$ consisting of homeomorphisms which take $D^{k}$ onto itself. Then

(iv) $\gamma: V^{k} \times V^{k} \rightarrow G_{0}^{k}\left(D^{n}\right) \subset G_{0}\left(D^{n}\right)$.

Now, let $W^{n-k}=\left\{y \in V^{n}, y_{i}=0,1 \leqq i \leqq k\right\}$. Then, there are natural projections $\pi_{1}: V^{n} \rightarrow V^{k}, \pi_{2}: V^{n} \rightarrow W^{n-k}$ given by

$$
\begin{aligned}
& \pi_{1}(y)=\pi_{1}\left(y_{1}, \cdots, y_{n}\right)=\left(y_{1}, \cdots, y_{k}\right), \\
& \pi_{2}(y)=\pi_{2}\left(y_{1}, \cdots, y_{n}\right)=\left(y_{k+1}, \cdots, y_{n}\right),
\end{aligned}
$$

$y \in V^{n}$. Define a map $\xi_{n, k}: V^{n} \rightarrow V^{n}$ by

$$
\xi_{n, k}(y)=\gamma\left(0, \pi_{1}(y)\right)\left(\pi_{2}(y)\right), \quad y \in V^{n} .
$$

Proposition (3.4). $\xi_{n, k} \sim 1:\left(V^{n}, V^{n}-0\right) \rightarrow\left(V^{n}, V^{n}-0\right)$ and the origin 0 remains stable during the connecting homotopy.

Proof. First observe that indeed $\xi_{n, k}\left(V^{n}-0\right) \subset\left(V^{n}-0\right)$. By direct computation (using the explicit formulas for $h_{n}$ and $g_{n}$ above)

$$
\xi_{n, k}(y)=A(y) \pi_{1}(y)+B(y) \pi_{2}(y),
$$

where $A$ and $B$ are continuous positive real functions on $V^{n}$. If $y \in V^{n}-0$, then the line segment joining $\xi_{n, k}(y)$ and $y$ necessarily avoids the origin and provides the required homotopy.

Suppose now that $M$ is an $n$-manifold and let $U$ denote an open set in $M$ whose closure is homeomorphic to $D^{n}$ with $V^{n}$ corresponding to $U$. Using part of the above material, there is a map

$$
\gamma: U \times U \rightarrow G(M),
$$

where $G(M)$ is the space of homeomorphisms of $M$ (c-o topology), such that

(i) $\gamma(x, y)(x)=y,(x, y) \in U \times U$;

(ii) $\gamma(x, x)=1, x \in U$;

(iii) $\gamma(x, y) \mid M-U=1,(x, y) \in U \times U$.

If $G_{0}(\bar{U})$ denotes the space of homeomorphisms of $\bar{U}$ which are fixed on on $\partial \bar{U}$, then $G_{0}(\bar{U})$ is a topological group naturally imbedded in $G(M)$, since $\bar{U}$ is compact. Hence if $i: G(M) \rightarrow G(M)$ is given by $i(h)=h^{-1}$, the function $i_{\gamma}: U \times U \rightarrow G(M)$ will be a map (i.e., continuous) even though $M$ is not compact.

We are now in a position to give some examples.

Proposition (3.5). Let $M$ denote an n-manifold, $\Delta$ the diagonal in $M \times M$ and $\pi: M \times M \rightarrow M$ projection on the first factor. Then

$$
\left(\mathscr{M}, \mathscr{M}_{0}\right)=(M \times M, M \times M-\Delta, \pi, M)
$$

is an $n$-hpb and in fact a locally trivial pair with fiber $\left(M, M-b_{0}\right), b_{0} \in M$. 
Proof. Let us first observe that we merely have to show triviality at a fixed point $b_{0} \in M$. Choose an open set $U$ such that $b_{0} \in U$ and $\bar{U}$ is homeomorphic to $D^{n}$. The local product structure at $b_{0}$ is given by

$$
\left(\pi^{-1}(U), \pi_{0}^{-1}(U)\right) \stackrel{\phi_{U}}{\longleftarrow}\left(U \times M, U \times\left(M-b_{0}\right)\right),
$$

where $\phi_{U}(b, y)=\left(b, \gamma\left(b_{0}, b\right)(y)\right)$. $\phi_{U}$ is a homeomorphism since $\phi_{U}^{-1}$, given by $\phi_{U}^{-1}(b, y)=\left(b,\left[\gamma\left(b_{0}, b\right)\right]^{-1}(y)\right)$, is a map by our above remarks on the map $i \gamma$.

REMARK (3.6). If $M$ is compact, $G(M)$ is a topological group. If $G_{0}(M)$ is the subgroup of $G(M)$ leaving $b_{0}$ fixed, then $G_{0}(M)$ acts (effectively) on the fiber $\left(M, M-b_{0}\right)$. It is a simple matter to show that in this case both $\mathscr{M}$ and $\mathscr{M}_{0}$ are in fact Steenrod bundles with group $G_{0}(M)$.

REMARK (3.7). $\left(\mathscr{K}, \mathscr{M}_{0}\right)$ is essentially the tangent microbundle of $M$ in the sense of Milnor.

Again, let $M$ denote an $n$-manifold and $T_{0} \subset M^{I}$ denote those paths $\omega$ such that $\omega(t)=\omega(0), 0 \leqq t \leqq 1$, if, and only if, $t=0$. Thus, $T_{0}$ are those paths which never return to their initial position (see Nash [10]). Furthermore, let $T$ denote $T_{0}$ plus all the constant paths in $M$. Define a map $p: T \rightarrow M$ by $p(\omega)=\omega(0), \omega \in T$.

Proposition (3.8). ( $\left.\mathscr{T}, \mathscr{T}_{0}\right)=\left(T, T_{0}, p, M\right)$ is an $n$-gpb and, in fact, a locally trivial pair with fiber $\left(F, F_{0}\right)$ having the same homotopy type as $\left(R^{n}, R^{n}-0\right)$.

Proof. The proof of the local product structure in $\left(\mathscr{T}, \mathscr{T}_{0}\right)$ is similar to that used in (2.10), employing the map $\gamma$. Let $\left(F, F_{0}\right)$ denote the fiber over a base point $b_{0} \in M$. Define $\sigma: M \rightarrow T$ by $\sigma(b)=\widetilde{b}$, the constant path at $b$. $\sigma$ is obviously a cross-section, and condition (i) for an $n$-gpb is satisfied. All that remains is to show that $\left(F, F_{0}\right) \sim\left(R^{n}, R^{n}-0\right)$. This is accomplished as follows. Let $U$ denote a Euclidean neighborhood of the base point $b_{0}$, and $\widetilde{U}=F \cap U^{I}, \widetilde{U}_{0}=F_{0} \cap U^{I}$. Then, by suitably shrinking paths (see [7], Theorem 7.2), $\left(\widetilde{U}, \widetilde{U}_{0}\right) \sim\left(F, F_{0}\right)$, where, in fact, the inclusion map $\left(\widetilde{U}, \widetilde{U}_{0}\right) \subset\left(F, F_{0}\right)$ is a homotopy equivalence. It suffices now to show that $\left(\widetilde{U}, \widetilde{U}_{0}\right) \sim\left(U, U-b_{0}\right)$. There is a $\operatorname{map} \beta:\left(\widetilde{U}, \widetilde{U}_{0}\right) \rightarrow\left(U, U-b_{0}\right)$ given by $\beta(\omega)$ $=\omega(1)$, and another map $\xi:\left(U, U-b_{0}\right) \rightarrow\left(\tilde{U}, \tilde{U}_{0}\right)$ given by letting $\xi(b)$ denote the line segment path from $b_{0}$ to $b$ (there is no loss of generality here in assuming $U=R^{n}$ and $\left.U-b_{0}=R^{n}-0\right)$. Clearly, $\beta \xi=1$ and it is a simple matter to show that $\xi \beta \sim 1:\left(\widetilde{U}, \widetilde{U}_{0}\right) \rightarrow\left(\widetilde{U}, \widetilde{U}_{0}\right)$ and our proof is complete.

Definition (3.9). ( $\mathscr{T}, \mathscr{T}_{0}$ ) (as in Proposition (3.8)) is called the tangent fiber space of the manifold $M$.

REMarK (3.10). Both Nash [10] and Hu [7] showed that $\left(T_{0}, p_{0}, M\right)$ was a fiber space in a sense strong enough to imply the universal covering 
homotopy theorem. Neither seemed to observe the local product structure.

REMARK (3.11). Let $G_{0}(M)$ denote the topological group in (3.5) when $M$ is compact. Then $G_{0}(M)$ acts on the fiber $\left(F, F_{0}\right)$ in $\left(\mathscr{T}, \mathscr{T}_{0}\right)$. Again, it is a simple matter to check that $\mathscr{T}$ and $\mathscr{T}_{0}$ are Steenrod bundles with group $G_{0}(M)$.

There is a natural map $\psi:\left(\mathscr{T}, \mathscr{T}_{0}\right) \rightarrow\left(\mathscr{K}, \mathscr{M}_{0}\right)$ given by

$$
\psi(\omega)=(\omega(0), \omega(1)) .
$$

When restricted to fibers,

$$
\psi_{0}=\psi \mid F:\left(F, F_{0}\right) \rightarrow\left(M, M-b_{0}\right)
$$

has the following property.

Proposition (3.12). $\psi_{0^{*}}: H_{*}\left(F, F_{0}\right) \rightarrow H_{*}\left(M, M-b_{0}\right)$ is an isomorphism.

Proof. Let $U$ denote a Euclidean neighborhood of $b_{0}$ such that the inclusion map $j:\left(U, U-b_{0}\right) \rightarrow\left(M, M-b_{0}\right)$ induces an isomorphism $j *$ : $H_{*}\left(U, U-b_{0}\right) \rightarrow H_{*}\left(M, M-b_{0}\right)$. It follows from the proof of (2.13) that the map $\xi:\left(U, U-b_{0}\right) \rightarrow\left(F, F_{0}\right)$, which assigns to $b \in U$ the line segment path from $b_{0}$ to $b$, is a homotopy equivalence. The commutativity of the following diagram completes the argument.

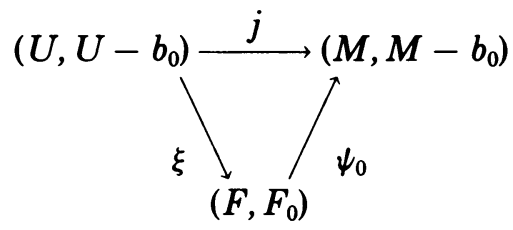

Proposition (3.13). If $G$ is any coefficient group, $\left(\mathscr{M}, \mathscr{M}_{0}\right)$ is G-orientable if, and only if, $\left(\mathscr{T}, \mathscr{T}_{0}\right)$ is G-orientable.

Proof. Let $\lambda^{\prime}, \lambda^{\prime \prime}$ denote lifting functions for $\left(\mathscr{T}, \mathscr{T}_{0}\right)$ and $\left(\mathscr{M}, \mathscr{M}_{0}\right)$, respectively, and let $\omega \in \Omega(B)$ denote a loop based at $b_{0}$. Define $\omega^{\prime}:\left(F, F_{0}\right)$ $\rightarrow\left(F, F_{0}\right)$ and $\omega^{\prime \prime}:\left(M, M-b_{0}\right) \rightarrow\left(M, M-b_{0}\right)$ by

$$
\begin{array}{ll}
\omega^{\prime}(y)=\lambda^{\prime}(y, \omega)(1), & y \in F, \\
\omega^{\prime \prime}(z)=\lambda^{\prime \prime}(z, \omega)(1), & z \in M .
\end{array}
$$

Then, the following diagram (using Proposition 1 of [12]) is homotopy commutative

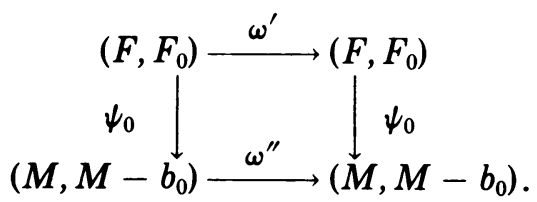

Hence, at the homology level $\omega_{*}^{\prime \prime} \psi_{0 *}=\psi_{0 *} \omega_{*}^{\prime}$ Since $\psi_{0 *}$ is an isomorphism, 
it follows that $\omega_{*}^{\prime \prime}$ is the identity homomorphism if, and only if, $\omega_{*}^{\prime}$ is. Thus, the action of $\Omega(B)$ on $H_{*}\left(F, F_{0} ; G\right)$ is trivial if, and only if, the action of $\Omega(B)$ on $H_{*}\left(M, M-b_{0} ; G\right)$ is trivial, and our result follows.

Definition (3.14). A manifold $M$ is orientable if the $n$-hpb $\left(\mathscr{K}, \mathscr{M}_{0}\right)$ is orientable.

REMARK (3.15). The above definition is justified since geometrically it means that when a generator $g$ of $H_{*}\left(M, M-b_{0}\right)$ is "carried" around a loop based at $b_{0}$, we "return" to $g$. It is easy to show that this definition is equivalent to others usually employed (e.g. the sheaf-theoretic definition or the definition of orientable in the triangulated case).

Proposition (3.16). A manifold $M$ is orientable if, and only if, its tangent fiber space $\left(\mathscr{T}, \mathscr{T}_{0}\right)$ is orientable as an $n$-gpb.

Suppose that the $n$-manifold $M$ possesses a differential structure and let $\mathscr{E}=(E, q, M)$ denote the tangent bundle of $M$ and $\mathscr{E}_{0}=\left(E, q_{0}, M\right), q_{0}$ $=q \mid E_{0}$, the sub-bundle of nonzero vectors. Then, $\left(\mathscr{E}_{\mathscr{E}}, \mathscr{E}_{0}\right)$ is clearly an $n$-gpb, in fact, the fiber is precisely $\left(R^{n}, R^{n}-0\right)$. It is known, Nash [10], that $\mathscr{E}_{0}$ is fiber homotopy equivalent to $\mathscr{T}_{0}$ and the same techniques show the following.

Proposition (3.17). ( $\left.\mathscr{T}, \mathscr{T}_{0}\right) \sim_{f}\left(\mathscr{E}, \mathscr{E}_{0}\right)$.

REmark (3.18). Theorem (2.5) may also be used to prove the above proposition.

Fibered pairs admit a "Whitney sum" as follows. Suppose $\left(\mathscr{F}, \mathscr{F}_{0}\right)$ $=\left(E, E_{0}, p, B\right),\left(\mathscr{F}^{\prime}, \mathscr{F}_{0}^{\prime}\right)=\left(E^{\prime}, E_{0}^{\prime}, p^{\prime}, B\right)$ are fibered pairs with respective fibers $\left(F, F_{0}\right)$ and $\left(F^{\prime}, F_{0}^{\prime}\right)$. Let

$$
E^{\prime \prime}=\left\{\left(x, x^{\prime}\right) \in E \times E^{\prime} \mid p(x)=p^{\prime}\left(x^{\prime}\right)\right\}
$$

and

$$
E_{0}^{\prime \prime}=\left[\left(E \times E_{0}^{\prime}\right) \cup\left(E_{0} \times E^{\prime}\right)\right] \cap E^{\prime \prime} .
$$

Then, we have the diagram (commutative)

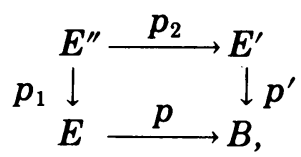

where $p_{1}$ and $p_{2}$ are projections on the first and second coordinates, respectively. Set $p^{\prime \prime}=p p_{1}=p^{\prime} p_{2}, p_{0}^{\prime \prime}=p^{\prime \prime} \mid E_{0}^{\prime \prime}, F^{\prime \prime}=F \times F^{\prime}$, and $F_{0}^{\prime \prime}=\left(F \times F_{0}^{\prime}\right)$ $\cup\left(F_{0} \times F^{\prime}\right)$.

Proposition-Definition (3.19). ( $\left.\mathscr{F}^{\prime \prime}, \mathscr{F}_{0}^{\prime \prime}\right)=\left\{E^{\prime \prime}, E_{0}^{\prime \prime}, p^{\prime \prime}, B\right\}$ is a fibered pair with fiber $\left(F^{\prime \prime}, F_{0}^{\prime \prime}\right)$ called the Whitney sum of $\left(\mathscr{F}, \mathscr{F}_{0}\right)$ and $\left(\mathscr{F}^{\prime}, \mathscr{F}_{0}^{\prime}\right)$ and is designated also by $\left(\mathscr{F}, \mathscr{F}_{0}\right) \oplus\left(\mathscr{F}^{\prime}, \mathscr{F}_{0}^{\prime}\right)$. 
Proof. Suppose $\lambda, \lambda^{\prime}$ are lifting functions for $\left(\mathscr{F}, \mathscr{F}_{0}\right)$ and $\left(\mathscr{F}^{\prime}, \mathscr{F}_{0}^{\prime}\right)$, respectively. A lifting function $\lambda^{\prime \prime}$ for $\left(\mathscr{F}^{\prime \prime}, \mathscr{F}_{0}^{\prime \prime}\right)$ is obtained by setting

$$
\lambda^{\prime \prime}\left(\left(x, x^{\prime}\right), \omega\right)(t)=\left(\lambda(x, \omega)(t), \lambda^{\prime}\left(x^{\prime}, \omega\right)(t)\right), \quad 0 \leqq t \leqq 1,
$$

where $\left(x, x^{\prime}\right) \in E^{\prime \prime}, \omega \in B^{I}$.

Remark (3.20). $\left(E^{\prime \prime}, p_{1}, E\right)$ is just the fiber space induced by the map $p: E \rightarrow B$ and the fiber space $\mathscr{F}^{\prime \prime}$ over $B$.

Proposition (3.21). If $\left(\mathscr{F}, \mathscr{F}_{0}\right)$ is an $n$-gpb and $\left(\mathscr{F}^{\prime}, \mathscr{F}_{0}^{\prime}\right)$ is a k-gpb, then their Whitney sum $\left(\mathscr{F}, \mathscr{F}_{0}\right) \oplus\left(\mathscr{F}^{\prime}, \mathscr{F}_{0}^{\prime}\right)$ is an $(n+k)$-gpb.

Proof. A simple exercise.

4. The normal fiber space. Suppose $M \subset S$ is a locally flat imbedding of the $n$-manifold $M$ in an $(n+k)$-manifold $S$. Let $N_{0}$ denote those paths $\omega \in S^{I}$ such that $\omega(t) \in M$ if, and only if, $t=0$. Let $N$ denote $N_{0}$ together with all the constant paths on $M$. Define a map $q: N \rightarrow M$ by setting $q(\omega)$ $=\omega(0), \omega \in N$.

Proposition (4.1). $\left(N_{,}, \mathscr{N}_{0}\right)=\left(N, N_{0}, q, M\right)$ is a k-gpb and, in fact, a locally trivial pair with fiber $\left(F^{\prime}, F_{0}^{\prime}\right)$ having the same homotopy type as $\left(R^{k}, R^{k}-0\right)$.

Proof. Let $D^{n+k}$ denote the closed unit $(n+k)$-ball in $R^{n+k}$ and $D^{n}$ the sub-ball of dimension $n$ given by those points of $D^{n+k}$ with last $k$ coordinates 0 . Let $G_{0}^{n}\left(D^{n+k}\right)$ denote the space of homeomorphisms (c-o topology) of $D^{n+k}$ which carry $D^{n}$ onto $D^{n}$. Then, if $V^{n+k}$ is the interior of $D^{n+k}$ and $V^{n}=V^{n+k} \cap D^{n}$, there is a map (see $\S 3$ )

$$
\gamma: V^{n} \times V^{n} \rightarrow G_{0}^{n}\left(D^{n+k}\right)
$$

such that

(i) $\gamma(x, y)(x)=y,(x, y) \in V^{n} \times V^{n}$;

(ii) $\gamma(x, x)=1, x \in V^{n}$;

(iii) $\gamma(x, y) \mid \partial D^{n+k}=1,(x, y) \in V^{n} \times V^{n}$.

Now, if $b_{0} \in M$, we may identify $D^{n+k}$ with a closed neighborhood (in $S$ ) of $b_{0}$ such that $D^{n+k} \cap M=D^{n}$, since $M \subset S$ is a locally flat imbedding. Then, $G_{0}^{n}\left(D^{n+k}\right)$ is a topological group naturally imbedded in $G(S)$, the space of homeomorphisms of $S$. Hence, if $i: G(S) \rightarrow G(S)$ is given by $i(h)$ $=h^{-1}$, the function $i_{\gamma}: V^{n} \times V^{n} \rightarrow G(S)$ is a map, without assuming $S$ compact. The local product structure of $\left(\mathscr{N}, \mathscr{N}_{0}\right)$ at $b_{0}$ is given as follows. Let $\left(F^{\prime}, F_{0}^{\prime}\right)=\left(q^{-1}\left(b_{0}\right), q_{0}^{-1}\left(b_{0}\right)\right)$, where, of course, $q_{0}=q \mid N_{0}$. Define

$$
\left(q^{-1}\left(V^{n}\right), q_{0}^{-1}\left(V^{n}\right)\right) \stackrel{\phi_{V^{n}}}{\longleftarrow}\left(V^{n} \times F^{\prime}, V^{n} \times F_{0}^{\prime}\right)
$$

by $\phi_{V^{n}}(b, \omega)(t)=\gamma\left(b_{0}, b\right)(\omega(t)), \quad 0 \leqq t \leqq 1, \quad(b, \omega) \in V^{n} \times F^{\prime} . \phi_{V^{n}}$ is a homeomorphism with $\phi_{V^{n}}^{-1}$ given by $\phi_{V^{n}}^{-1}(\omega)=\left(\omega(0), \omega^{\prime}\right)$, with $\omega^{\prime}(t)=(i \gamma)(\omega(t))$, 
$0 \leqq t \leqq 1$. Since $b_{0}$ is arbitrary and since for any other point $b_{1} \in M$, we have $\left(q^{-1}\left(b_{1}\right), q_{0}^{-1}\left(b_{1}\right)\right)$ homeomorphic to $\left(F^{\prime}, F_{0}^{\prime}\right),\left(\mathcal{N}, \mathscr{N}_{0}\right)$ is a locally trivial fibered pair.

Now, the cross-section $\sigma^{\prime}: M \rightarrow N$, required of a $k$-gpb is obtained by assigning as $\sigma^{\prime}(b)$ the constant path at $b$ and obviously $N_{0}=N-\sigma^{\prime}(M)$.

It remains to show that $\left(F^{\prime}, F_{0}^{\prime}\right) \sim\left(R^{k}, R^{k}-0\right)$. Let $V^{n+k}=V$ denote the neighborhood in $S$ of $b_{0}$ employed above and let $\widetilde{V}=F^{\prime} \cap V^{I}, \widetilde{V}_{0}$ $=F_{0}^{\prime} \cap V^{I}$. By suitably shrinking paths (again see [11, Theorem 7.2]), $\left(\widetilde{V}, \widetilde{V}_{0}\right) \sim\left(F^{\prime}, F_{0}^{\prime}\right)$ with the inclusion $\left(\widetilde{V}, \widetilde{V}_{0}\right) \subset\left(F^{\prime}, F_{0}^{\prime}\right)$ giving the homotopy equivalence. Let $W=V^{n}=V \cap D^{n}$ as above and observe that $(V, W)$ $=\left(R^{n+k}, R^{n}\right)$ and hence

$$
\left((V-W) \cup b_{0}, V-W\right)=\left(\left(R^{n+k}-R^{n}\right) \cup 0, R^{n+k}-R^{n}\right) \sim\left(R^{k}, R^{k}-0\right) .
$$

Furthermore, there is a map $\beta:\left(\widetilde{V}, \widetilde{V}_{0}\right) \rightarrow\left((V-W) \cup b_{0}, V-W\right)$ given by $\beta(\omega)=\omega(1)$ and another map $\xi:\left((V-W) \cup b_{0}, V-W\right) \rightarrow\left(\widetilde{V}, \widetilde{V_{0}}\right)$ given by letting $\xi(b)$ denote the line segment path from $b_{0}$ to $b$. Clearly $\beta \xi=1$ and (just as in Proposition (3.7)) it is a simple matter to check that $\xi \beta \sim 1$. Thus, $\left(F^{\prime}, F_{0}^{\prime}\right) \sim\left(R^{k}, R^{k}-0\right)$ and our proof is complete.

REMARK (4.2). Suppose $S$ (as above) is compact, and let $G_{0}^{M}(S)$ denote the subgroup of $G(S)$ consisting of homeomorphisms $h$ leaving the base point $b_{0}$ fixed and such that $h(M)=M$. Then, $G_{0}^{M}(S)$ is a topological group and one checks easily that $\mathscr{N}$ and $\mathscr{N}_{0}$ are Steenrod bundles with group $G_{0}^{M}(S)$.

Definition (4.3). The $k$-gpb $\left(\mathcal{N}, \mathscr{N}_{0}\right)$ of Proposition (4.1) is called the normal fiber space to the locally flat imbedding $M \subset S$.

Suppose $M \subset S$ as above.

DEFinition (4.4). A tubular neighborhood of $M$ is an open set $U \supset M$ which admits a map $p: U \rightarrow M$ such that the inclusion $i: M \rightarrow U$ is a crosssection for $p$ and if $U_{0}=U-M$, then $\left(U, U_{0}, p, M\right)$ is a locally trivial fibered pair with fiber $\left(R^{k}, R^{k}-0\right)$. Furthermore, we assume the existence of a homotopy $H: U \times I \rightarrow U$ such that

(i) $p(H(x, t))=p(x), 0 \leqq t \leqq 1, x \in U$;

(ii) $H(x, t)=x, x \in M, 0 \leqq t \leqq 1$;

(iii) $H(x, t) \in U_{0}, x \in U_{0}, 0 \leqq t \leqq 1$;

(iv) $H_{0}=p, H_{1}=1$.

REMARK (4.5). It is clear that if $M \subset S$ admits a tubular neighborhood, then $M$ is necessarily locally flat in $S$. The converse is an open question and appears perhaps difficult and certainly interesting.

REMARK (4.6). It is likely that in Definition (4.4) the existence of the homotopy $H$ is a consequence of the other conditions required of a tubular neighborhood.

REMARK (4.7). If $M$ possesses a differential structure and if $M \subset R^{n+k}$ is a differentiable imbedding, then $M$ admits (via the classical normal $k$ plane bundle) a tubular neighborhood $U$. 
Proposition (4.8). If $\left(U, U_{0}, p, M\right)$ is a tubular neighborhood of $M \subset S$ (as above), then $\left(U, U_{0}, p, M\right) \sim{ }_{f}\left(\mathscr{N}, \mathscr{N}_{0}\right)$, the normal fiber space of the imbedding.

Proof. Let $H: U \times I \rightarrow U$ denote the homotopy in Definition (4.4) associated with the normal neighborhood $U$. Then, $H$ may be interpreted as a map $\widetilde{H}: U \rightarrow U^{I}$. Note that if $x \in M, \widetilde{H}(x)$ is the constant path at $x$, and if $x \in U_{0}, \widetilde{H}(x)$ is a path in $U$ (hence in $S$ ) which begins at $p(x) \in M$, ends at $x$ and $\widetilde{H}(x)(t) \in M$ if, and only if, $t=0$. Hence, $x \in U_{0}$ implies $\widetilde{H}(x) \in N_{0}$. Thus we have a map of fibered pairs

$$
\widetilde{H}:\left(U, U_{0}, p, M\right) \rightarrow\left(\mathcal{N}, \mathscr{N}_{0}\right) .
$$

It is a simple matter to check that on a single fiber $\widetilde{H}$ is a homotopy equivalence of pairs and hence $\widetilde{H}$ is also a fiber homotopy equivalence, using Theorem (2.5).

Corollary (4.9). Suppose $M \subset R^{n+k}$ is a differentiable imbedding of the differentiable n-manifold $M$ and $\left(N^{\prime}, N_{0}^{\prime}\right)=\left(N^{\prime}, N_{0}^{\prime}, q^{\prime}, M\right)$ is the classical normal $k$-plane bundle written as a fibered pair with $N^{\prime}$ the space of normal vectors, $N_{0}^{\prime}$ the space of nonzero normal vectors and $q^{\prime}$ assigns to each vector its initial point. Then $\left(\mathscr{N}^{\prime}, \mathscr{N}_{0}^{\prime}\right) \sim{ }_{f}\left(\mathcal{N}, \mathscr{N}_{0}\right)$.

Proof. This is an immediate consequence of Remark (4.7) and Proposition (4.8).

Definition (4.10). If $\left(\mathscr{F}, \mathscr{F}_{0}\right)=\left(E, E_{0}, p, B\right)$ is a fibered pair and $A \subset B$ is a subspace of $B$, then $\left(\mathscr{F}, \mathscr{F}_{0}\right) \mid A$, the restriction of $\left(\mathscr{F}, \mathscr{F}_{0}\right)$ to $A$, is the fibered pair $\left(p^{-1}(A), p_{0}^{-1}(A), p \mid p^{-1}(A), A\right)$.

We now reach the fundamental theorem of this section.

THEOREM (4.11). Suppose $M \subset S$ is a locally flat imbedding of an n-manifold $M$ in an $(n+k)$-manifold $S$. Suppose further that $\left(\mathscr{T}, \mathscr{T}_{0}\right)$ is the tangent fiber space of $M,\left(\mathscr{N}, \mathscr{N}_{0}\right)$ is the normal fiber space associated with the imbedding and $\left(\mathscr{T}^{*}, \mathscr{T}_{0}^{*}\right)$ the tangent fiber space of $S$. Then

$$
\left(\mathscr{T}, \mathscr{T}_{0}\right) \oplus\left(\mathscr{N}, \mathscr{N}_{0}\right) \sim_{f}\left(\mathscr{T}^{*}, \mathscr{T}_{0}^{*}\right) \mid M .
$$

Before we proceed with the proof, we will set forth a few lemmas, assuming the hypotheses and notation of Theorem (4.11) as well as the following notation.

$$
\begin{aligned}
& \left(\mathscr{T}, \mathscr{T}_{0}\right)=\left(T, T_{0}, p, M\right), \quad \text { fiber }\left(F, F_{0}\right) \text {; } \\
& \left(\mathcal{N}, \mathscr{N}_{0}\right)=\left(N, N_{0}, q, M\right), \quad \text { fiber }\left(F^{\prime}, F_{0}^{\prime}\right) \text {; } \\
& \left(\mathscr{T}^{*}, \mathscr{T}_{0}^{*}\right) \mid M=\left(\overline{\mathscr{T}}, \overline{\mathscr{T}}_{0}\right)=\left(\bar{T}, \bar{T}_{0}, \bar{p}, M\right), \quad \text { fiber }\left(\bar{F}, \bar{F}_{0}\right) ; \\
& \left(\mathscr{T}, \mathscr{T}_{0}\right) \oplus\left(\mathcal{N}, \mathscr{N}_{0}\right)=\left(E^{\prime \prime}, E_{0}^{\prime \prime}, q^{\prime \prime}, M\right) \text {, fiber }\left(F^{\prime \prime}, F_{0}^{\prime \prime}\right) \text {. }
\end{aligned}
$$

Lemma (4.12). There is an open covering $\left\{U_{\alpha}\right\}$ of $M$ and a local product structure for $\left(\overline{\mathscr{T}}, \overline{\mathscr{T}}_{0}\right)$ 


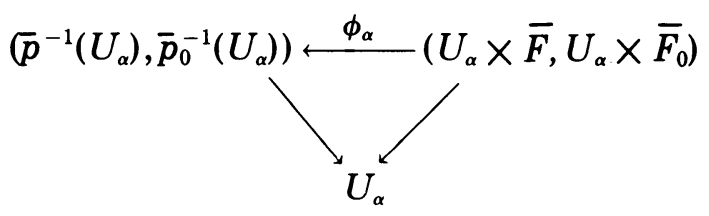

such that

(a) If $\omega_{0}$ is the unique constant path in $\bar{F}, \phi_{\alpha}\left(b, \omega_{0}\right)$ is the constant path at $b$.

(b) $\phi_{\alpha}\left(U_{\alpha} \times F_{0}^{\prime}\right) \subset N_{0}$.

(c) Hence, $\phi_{\alpha}$ (properly restricted) also provides a local product structure for $\left(\mathcal{N}, \mathscr{N}_{0}\right)$.

Proof. Because the situation is homogeneous, we restrict ourselves to a fixed $b_{0} \in M$, with $b_{0}$ also determining the respective fibers. Employing the notation of Proposition (4.1), we may identify $D^{n+k}$ with a closed neighborhood (in $S$ ) of $b_{0}$ such that $D^{n+k} \cap M=D^{n}$. Recall from $\$ 3$ the map

$$
\gamma: V^{n+k} \times V^{n+k} \longrightarrow G_{0}\left(D^{n+k}\right)
$$

which also satisfies

$$
\gamma: V^{n} \times V^{n} \longrightarrow G_{0}^{n}\left(D^{n+k}\right) .
$$

The local product structure for $\left(\mathscr{T}^{*}, \mathscr{T}_{0}^{*}\right)$, the tangent fiber space of $S$ is given (Proposition (3.8)) as follows:

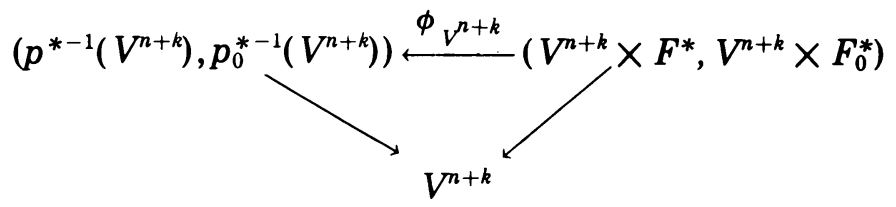

where $\phi V^{n+k}(b, \omega)(t)=\gamma\left(b_{0}, b\right)(\omega(t)), 0 \leqq t \leqq 1, \omega \in F^{*}$. A look at the proof of Proposition (4.1) tells us that $\phi_{V^{n+k}}$ restricted to $\left(V^{n} \times F^{\prime}, V^{n} \times F_{0}^{\prime}\right)$ is precisely $\phi_{V^{n}}$ in that proposition. Since $\left(F^{*}, F_{0}^{*}\right)=\left(\bar{F}, \bar{F}_{0}\right), \phi_{V^{n+k}}$ restricted to $\left(V^{n} \times \bar{F}, V^{n} \times \bar{F}_{0}\right)$ gives a local product structure at $b_{0}$ satisfying condition (b) of the lemma. Condition (a) is easily observable and our lemma follows.

Lemma (4.13). There is a lifting function $\lambda$ for the fibered pair $\left(\overline{\mathscr{T}}, \overline{\mathscr{T}}_{0}\right)$ with the following properties.

(a) If $\omega_{0}$ is a constant path in $M$ ( hence $\omega_{0} \in \bar{T}$ ) then for any path $\alpha$ in $M$ such that $\omega_{0}(0)=\alpha(0)$, we have $\lambda\left(\omega_{0}, \alpha\right)(t)$ is the constant path at $\alpha(t)$, $0 \leqq t \leqq 1$.

(b) If $\omega_{0} \in N_{0}$ and $\alpha$ is a path in $M$ such that $\omega_{0}(0)=\alpha(0)$, then $\lambda\left(\omega_{0}, \alpha\right)(t)$ $\in N_{0}, 0 \leqq t \leqq 1$.

Proof. Apply the proof of the Hurewicz Uniformization Theorem [8] (M is paracompact) to an open covering of $M$ with the properties indicated in Lemma (4.12). 
Lemma (4.14). Suppose $\left(\mathscr{F}, \mathscr{F}_{0}\right)=\left(E, E_{0}, p, B\right)$ is a locally trivial fibered pair, fiber $\left(F, F_{0}\right)$, with a given open covering $\left\{U_{\alpha}\right\}$ and local product structures

$$
\left(p^{-1}\left(U_{\alpha}\right), p_{0}^{-1}\left(U_{\alpha}\right)\right) \stackrel{\phi_{\alpha}}{\longleftarrow}\left(U_{\alpha} \times F, U_{\alpha} \times F_{0}\right) .
$$

Suppose $U_{0}$ is a member of $\left\{U_{\alpha}\right\}$ containing the base point $b_{0}$ and $\phi_{0}$ the homeomorphism giving the local product structure over $U_{0}$. Then, for any open set $V$ in $B$ such that $b_{0} \in V \subset \bar{V} \subset U_{0}$, there is a lifting function $\lambda$ for $\left(\mathscr{F}, \mathscr{F}_{0}\right)$ with the property that if $x \in F$ and $\omega$ is a path in $V$ such that $p(x)=\omega(0)$, then

$$
\lambda(x, \omega)(t)=\phi_{0}(\omega(t), x), \quad 0 \leqq t \leqq 1 .
$$

Proof. Take the covering $\left\{U_{\alpha}\right\}$ and obtain a refinement as follows. $\underline{U}_{0}$ and $V$ are given so that $b_{0} \in V \subset \bar{V} \subset U_{0}$. For $U_{\alpha} \neq U_{0}$ let $V_{\alpha}=(B-\bar{V})$ $\cap U_{\alpha}$. Then $\left\{V_{\alpha}, U_{0}\right\}$ is a refinement of $\left\{U_{\alpha}\right\}$ with only $U_{0}$ covering points of $\bar{V}$. Now, apply the proof of the Uniformization Theorem of Hurewicz [8] to the open cover $\left\{V_{\alpha}, U_{0}\right\}$.

Lemma (4.15). There is a lifting function $\lambda$ for the fibered pair $\left(\overline{\mathscr{T}}, \overline{\mathscr{T}}_{0}\right)$ satisfying the three conditions of Lemma (4.13) and in addition the following. If $D^{n+k}$ is a closed neighborhood of the base point $b_{0}$, as in Lemma (4.12), with interior $V^{n+k}$, then for some subset $W$ (open in $M$ ) of $V^{n}=V^{n+k} \cap M$,

$$
\lambda(\omega, \alpha)(t)(s)=\phi_{V^{n+k}}(\alpha(t), \omega)(s)=\gamma\left(b_{0}, \alpha(t)\right) \omega(s), \quad 0 \leqq s, t \leqq 1,
$$

where $\omega \in \bar{F}, \alpha \in W^{I}$, and $\omega(0)=\alpha(0)$.

Proof. Combine Lemmas (4.13) and (4.14).

We turn to the proof of Theorem (4.11).

Proof of Theorem (4.11). Let $\lambda$ denote a lifting function for $\left(\overline{\mathscr{T}}, \overline{\mathscr{T}}_{0}\right)$ satisfying the conditions of Lemma (4.15). A map of fibered pairs

$$
\psi:\left(\mathscr{T}, \mathscr{T}_{0}\right) \oplus\left(\mathcal{N}, \mathscr{N}_{0}\right) \rightarrow\left(\overline{\mathscr{T}}, \overline{\mathscr{T}}_{0}\right)
$$

is obtained by setting

$$
\psi(\sigma, \tau)(t)=\lambda(\tau, \sigma)(t)(t), \quad 0 \leqq t \leqq 1 .
$$

Note that $\bar{p}(\tau)=\tau(0)=\sigma(0)$, since $(\sigma, \tau) \in E^{\prime \prime}$ and the application of $\lambda$ is permissible. If the path $\tau \in N_{0}$, then the path $\lambda(\tau, \sigma)(t) \in N_{0_{2}} 0 \leqq t \leqq 1$. Hence for $t>0, \lambda(\tau, \sigma)(t)(t) \notin M$ and thus a fortiori, $\psi(\sigma, \tau) \in \bar{T}_{0}$. If $\sigma \in T_{0}$ and $\tau \in N$ is a constant path $\psi(\sigma, \tau)=\sigma \in T_{0} \subset \bar{T}_{0}$. Thus, if $(\sigma, \tau) \in E_{0}^{\prime \prime}$, $\psi(\sigma, \tau) \in \bar{T}_{0}$. If $(\sigma, \tau)$ consists of constant paths, $\psi(\sigma, \tau)$ is likewise the constant paths at $\sigma(0)=\tau(0)$. Clearly $\bar{p} \psi(\sigma, \tau)=q^{\prime \prime}(\sigma, \tau)$ and $\psi$ is then a welldefined map of fibered pairs. All that remains is to show that the restriction of $\psi$ to a fiber, namely,

$$
\psi_{0}:\left(F^{\prime \prime}, F_{0}^{\prime \prime}\right)=\left(F \times F^{\prime},\left(F \times F_{0}^{\prime}\right) \cup\left(F_{0} \times F^{\prime}\right)\right) \rightarrow\left(\bar{F}, \overline{F_{0}}\right)
$$


is an ordinary homotopy equivalence. Let $b_{0}$ denote our base point and $D^{n+k}$ a closed neighborhood in $S$ of $b_{0}$ as in Lemma (4.15), with interior $V^{n+k}$. Then there is a smaller open $(n+k)$-ball $W^{n+k} \subset V^{n+k}$ such that $W^{n}$ $=W^{n+k} \cap M$ has the properties also given in Lemma (4.15) with $W$ identified with $W^{n}$. Let $W^{k}$ denote those points of $W^{n+k}$ whose first $n$ coordinates are 0 and let $\pi_{1}: W^{n+k} \rightarrow W^{n}, \pi_{2}: W^{n+k} \rightarrow W^{k}$ denote the natural projections on the first $n$ and last $k$ coordinates, respectively. Furthermore, if $x \in W^{n+k}$, let $\omega(x)$ denote the line segment path from 0 to $x$. Define maps

$$
\begin{aligned}
& \alpha:\left(W^{n+k}, W^{n+k}-0\right) \rightarrow\left(F^{\prime \prime}, F_{0}^{\prime \prime}\right), \\
& \beta:\left(W^{n+k}, W^{n+k}-0\right) \rightarrow\left(\bar{F}, \bar{F}_{0}\right)
\end{aligned}
$$

by

$$
\alpha(x)=\left(\omega\left(\pi_{1}(x)\right), \omega\left(\pi_{2}(x)\right)\right), \quad \beta(x)=\omega(x),
$$

$x \in W^{n+k}$. By previous arguments (Propositions (3.8), (3.21), (4.1)) $\alpha$ and $\beta$ are homotopy equivalences. Consider, finally, the diagram

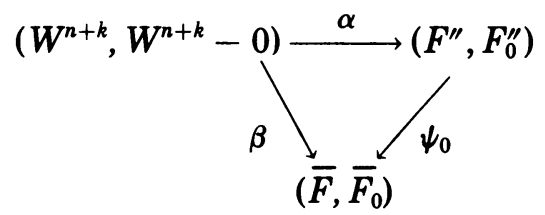

$\psi_{0}$ will be a homotopy equivalence provided we can show the diagram is homotopy commutative. Take $x \in W^{n+k}$ and $0 \leqq t \leqq 1$. By Lemma (4.15)

$$
\begin{aligned}
\psi_{0}(\alpha(x))(t) & =\lambda\left(\omega\left(\pi_{2}(x)\right), \omega\left(\pi_{1}(x)\right)\right)(t)(t) \\
& =\gamma\left(b_{0}, t \pi_{1}(x)\right)\left(\omega\left(\pi_{2}(x)\right)(t)\right) \\
& =\gamma\left(b_{0}, t \pi_{1}(x)\right)\left(t \pi_{2}(x)\right) \\
& =\xi_{n+k, n}(t x),
\end{aligned}
$$

where $\xi_{n+k, n}$ is the map of Proposition (3.4), with $b_{0}$ identified with 0 . On the other hand $\beta(x)(t)=t x$. By Proposition (3.4) $\xi_{n+k, n} \sim 1:\left(W^{n+k}, W^{n+k}-0\right)$ $\rightarrow\left(W^{n+k}, W^{n+k}-0\right)$. Consider a homotopy

$$
H:\left(W^{n+k}, W^{n+k}-0\right) \times I \rightarrow\left(W^{n+k}, W^{n+k}-0\right)
$$

such that $H_{0}=1$ and $H_{1}=\xi_{n+k, n}$. Recall that $H$ keeps the origin fixed during the homotopy. Using $H$, we can define a homotopy

$$
G:\left(W^{n+k}, W^{n+k}-0\right) \times I \rightarrow\left(\bar{F}, \bar{F}_{0}\right)
$$

by

$$
G(x, s)(t)=H(t x, s), x \in W^{n+k}, \quad 0 \leqq s \leqq t \leqq 1 .
$$

It is clear that $G_{0}=\beta$ and $G_{1}=\psi_{0} \alpha$ and the diagram is homotopy commu- 
tative and hence $\psi_{0}$ is a homotopy equivalence. Thus our proof of Theorem (4.11) is complete.

5. The Thom isomorphism. Let us first recall [1] the following theorem which we state for cohomology.

THEOREM (5.1). Let $\left(\mathscr{F}, \mathscr{F}_{0}\right)=\left(E, E_{0}, p, B\right)$ denote a fibered pair with fiber $\left(F, F_{0}\right)$. Then, there is a spectral sequence associated with a filtration of singular cochains $C^{*}\left(E, E_{0} ; G\right), G$ any coefficient group, whose $E_{2}$-term is naturally isomorphic to $H^{*}\left(B, H^{*}\left(F, F_{0} ; G\right)\right.$ ), where $H^{*}\left(F, F_{0} ; G\right)$ is a local coefficient system determined by the action of $\Omega(B)$ on $H^{*}\left(F, F_{0} ; G\right)$ as given in $\S 2$.

Let us denote the terms of the spectral sequence associated with the fibered pair $\left(\mathscr{F}, \mathscr{F}_{0}\right)$ by $\bar{E}_{r}^{p, q}$ and the terms of the spectral sequence associated with the single fibration $\mathscr{F}=(E, p, B)$ by $E_{r}^{p, q}$. Let us suppose that our coefficient group $G$ is a commutative ring $\Lambda$ with unit. Then, following the techniques in Serre [12], a mixed cup product of the type $H^{*}(X: \Lambda)$ $\otimes H^{*}(X, A ; \Lambda) \rightarrow H^{*}(X, A ; \Lambda)$ can be introduced into these spectral sequences to induce pairings

$$
\smile: E_{r}^{p, q} \otimes \bar{E}_{r}^{p^{\prime}, q^{\prime}} \rightarrow \bar{E}_{r}^{p+p^{\prime}, q+q^{\prime}} .
$$

This cup product also behaves well with respect to the spectral sequence isomorphisms, i.e., the following diagram is com:nutative

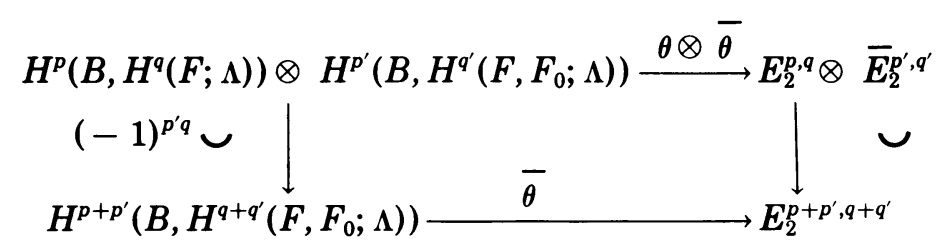

where $\theta: H^{p}\left(B, H^{q}(F ; \Lambda)\right) \rightarrow E_{2}^{p, q}$ and $\overline{\theta:} H^{p^{\prime}}\left(B, H^{q^{\prime}}\left(F, F_{0} ; \Lambda\right)\right) \rightarrow \bar{E}_{2}^{p^{\prime}, q^{\prime}}$ are the spectral sequence isomorphisms for $\mathscr{F}$ and $\left(\mathscr{F}, \mathscr{F}_{0}\right)$, respectively, and the cup product on the left is induced by the cup product

$$
H^{*}(F ; \Lambda) \otimes H^{*}\left(F, F_{0} ; \Lambda\right) \rightarrow H^{*}\left(F, F_{0} ; \Lambda\right) .
$$

ThEOREM 5.2 (Тном IsOMorphism). Let $\left(\mathscr{F}, \mathscr{F}_{0}\right)=\left(E, E_{0}, p, B\right)$ denote an $n$-hpb with fiber $\left(F, F_{0}\right)$ which is $\Lambda$-orientable and which has an acyclic total fiber, i.e., $H^{q}(F ; \Lambda)=0$ for $q>0$ and $H^{0}(F ; \Lambda)=\Lambda$. Then, there is an element $U \in H^{n}\left(E, E_{0} ; \Lambda\right)$ (corresponding to the unit in $\Lambda$ ) and a commutative diagram of isomorphisms, $j$ arbitrary,

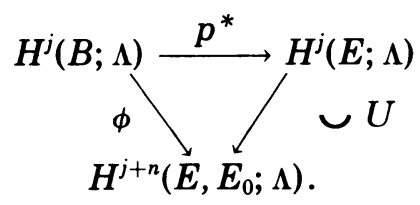

Proof. First, we observe the commutative diagram 


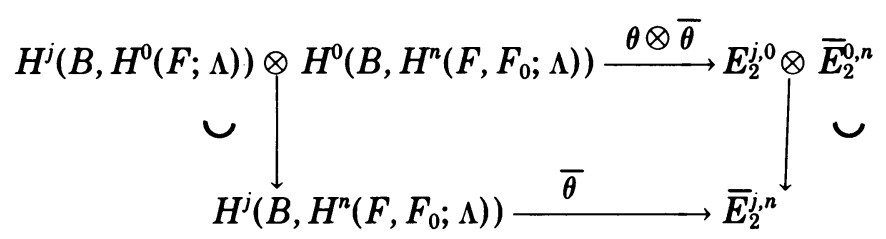

Since both spectral sequences are trivial and, in fact, $E_{\infty}^{j, q}=0$ for $q \neq 0$, $\bar{E}_{\infty}^{j, q}=0$ for $q \neq n$, we may replace the vertical homomorphism on the right by

$$
H^{j}(E ; \Lambda) \otimes H^{n}\left(E, E_{0} ; \Lambda\right) \stackrel{\smile}{\longrightarrow} H^{j+n}\left(E, E_{0} ; \Lambda\right) .
$$

Identifying $H^{0}(F ; \Lambda)$ and $H^{n}\left(F, F_{0} ; \Lambda\right)$ with $\Lambda$, we then obtain the commutative diagram

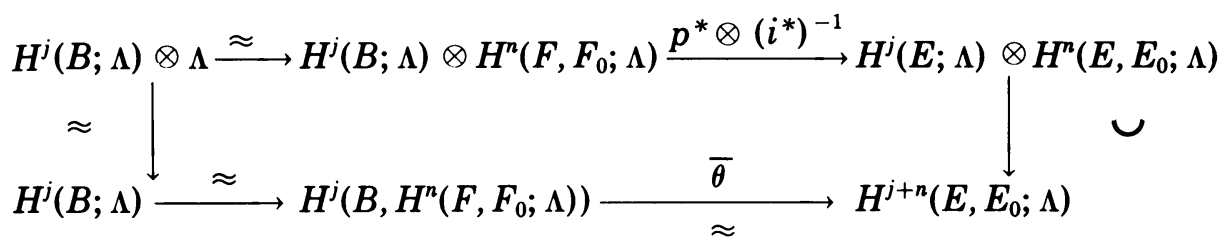

where the vertical isomorphism is the standard one $\left(\otimes=\otimes_{\Lambda}\right)$ and $p^{*} \otimes\left(i^{*}\right)^{-1}$ is readily identified, where $i:\left(F, F_{0}\right) \rightarrow\left(E, E_{0}\right)$ is the inclusion map. Standard arguments show that $p^{*}$ and $i^{*}$, are in fact, isomorphisms. Let $\bar{U}$ correspond to the unit of $\Lambda$ under the identification $\Lambda=H^{n}\left(F, F_{0} ; \Lambda\right)$ and let $U$ be chosen by $i^{*}(U)=\bar{U}$. Then, letting $\phi$ denote the composition of the lower horizontal homomorphisms in the above diagram, we have the desired result.

REMARK (5.3). Theorem (5.2) is valid, a fortiori, for $n$-gpb's.

6. Stiefel-Whitney classes. Now that a certain amount of fundamental theory of $n$-gpb's has been developed we rely strongly on the theory of Stiefel-Whitney classes already developed in the differentiable case. In particular, we follow the exposition of Milnor [9]. Throughout this section the coefficient group will be $Z_{2}$, when no coefficient group is displayed in the notation.

If $\left(\mathscr{F}, \mathscr{F}_{0}\right)=\left(E, E_{0}, p, B\right)$, with fiber $\left(F, F_{0}\right)$ is an $n$-gpb, we have the following diagram

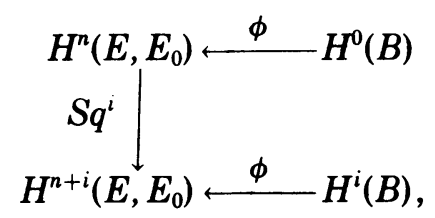

where $\phi$ is the Thom isomorphism of $\$ 5$, and $S q^{i}$ is the $i$ th Steenrod Square.

Definition $(6.1)$. If $\left(\mathscr{F}, \mathscr{F}_{0}\right)$ is an $n$-gpb, the $i$ th Stiefel-Whitney class $W_{i}\left(\mathscr{F}, \mathscr{F}_{0}\right)$ is defined by 


$$
W_{i}\left(\mathscr{F}, \mathscr{F}_{0}\right)=\phi^{-1} S q^{i} \phi(1)
$$

where 1 is the unit of the cohomology ring $H^{*}(B)$. The total Stiefel-Whitney class $W\left(\mathscr{F}, \mathscr{F}_{0}\right)$ is given by

$$
W\left(\mathscr{F}, \mathscr{F}_{0}\right)=1+W_{1}\left(\mathscr{F}, \mathscr{F}_{0}\right)+W_{2}\left(\mathscr{F}, \mathscr{F}_{0}\right)+\cdots+W_{n}\left(\mathscr{F}, \mathscr{F}_{0}\right) .
$$

REMARK (6.2). If $\left(\mathscr{F}, \mathscr{F}_{0}\right)$ is an $n$-gpb, then $W_{i}\left(\mathscr{F}, \mathscr{F}_{0}\right)=0$ for $i>n$.

Let $M$ denote a (topological) $n$-manifold and $\left(\mathscr{T}, \mathscr{T}_{0}\right)$ its tangent fiber space, which is an $n$-gpb.

Definition (6.3). $W_{i}(M)=W_{i}\left(\mathscr{T}, \mathscr{T}_{0}\right)$ is called the $i$ th Stiefel-Whitney class of $M . W(M)=W\left(\mathscr{T}, \mathscr{T}_{0}\right)$ is the total Stiefel-Whitney class of $M$.

If $M \subset S$, where $S$ is an $(n+k)$-manifold, is a locally flat imbedding, then we have an associated normal fiber space $\left(\mathscr{N}, \mathscr{N}_{0}\right)$ which is a $k$-gpb.

Definition (6.4). $\bar{W}_{i}(M)=W_{i}\left(\mathcal{N}, \mathscr{N}_{0}\right)$ is called the $i$ th dual StiefelWhitney class of $M . \bar{W}(M)=W\left(\mathcal{N}, \mathscr{N}_{0}\right)$ is the total dual Stiefel-Whitney class of $M . \bar{W}(M)$, of course, depends upon the imbedding $M \subset S$.

REMARK (6.5). We have yet to justify the term "dual" for $\bar{W}(M)$. This will be done shortly when we prove the Whitney Duality Theorem in this setting (\$6) which yields for the special case $M \subset R^{n+k}, W(M) \cup \bar{W}(M)=1$.

The following propositions are elementary.

Proposition (6.6). If two $n$-gpb's $\left(\mathscr{F}, \mathscr{F}_{0}\right)$ and $\left(\mathscr{F}^{\prime}, \mathscr{F}_{0}^{\prime}\right)$ are fiber homotopy equivalent, then $W_{i}\left(\mathscr{F}, \mathscr{F}_{0}\right)=W_{i}\left(\mathscr{F}^{\prime}, \mathscr{F}_{0}^{\prime}\right), 0 \leqq i \leqq n$.

Definition (6.7). The fibered pair $\left(B \times R^{n}, B \times\left(R^{n}-0\right), \pi_{1}, B\right)$ where $\pi_{1}$ is projection on $B$ is called the trivial $n$-gpb over $B$.

Proposition (6.8). If an $n$-gpb ( $\left.\mathscr{F}, \mathscr{F}_{0}\right)$ is fiber homotopy equivalent to the trivial $n$-gpb over the base for $\left(\mathscr{F}, \mathscr{F}_{0}\right)$, then $W\left(\mathscr{F}, \mathscr{F}_{0}\right)=1$.

Proposition (6.9). If an $n$-gpb $\left(\mathscr{F}, \mathscr{F}_{0}\right)$ has a contractible base, it is fiber homotopy equivalent to the trivial $n$-gpb.

Theorem (6.10). If $M$ possesses a differential structure, the classes $W_{i}(M)$ are the classical Stiefel-Whitney classes [9]. Furthermore, if $M$ is differentiably imbedded in $R^{n+k}$, the classes $\bar{W}_{i}(M)$ coincide with the classical dual Stiefel-Whitney classes [9].

Proof. This is an immediate consequence of Propositions (3.16), (4.4) and (6.7).

Theorem (6.11). If $\left(\mathscr{F}, \mathscr{F}_{0}\right)$ is an $n$-gpb and $\left(\mathscr{F}^{\prime}, \mathscr{F}_{0}^{\prime}\right)$ is a $k$-gpb over the same base $B$ and if $\left(\mathscr{F}^{\prime \prime}, \mathscr{F}_{0}^{\prime \prime}\right)$ is their Whitney sum, then

$$
W\left(\mathscr{F}^{\prime \prime}, \mathscr{F}_{0}^{\prime \prime}\right)=W\left(\mathscr{F}, \mathscr{F}_{0}\right) \smile W\left(\mathscr{F}^{\prime}, \mathscr{F}_{0}^{\prime}\right)
$$

where multiplication on the right is cup product in $H^{*}(B)$.

Proof. The proof is essentially word for word the argument in Milnor 
[9] with the following comment supplied. If $p: E \rightarrow B$ is an $n$-plane bundle in the usual sense then $p$ is a homotopy equivalence. In fact if $\sigma: B \rightarrow E$ is the 0 -cross-section, then $\sigma p \sim 1$ in a fiberwise manner, the homotopy being supplied by scalar multiplication in $E$. On the other hand, if $p: E \rightarrow B$ is a Hurewicz fibration with contractible fiber, $p$ need not be a homotopy equivalence. Nevertheless, using the Vietoris Mapping Theorem for fiber spaces and singular homology (Serre [12]), $p$ induces isomorphisms at the cohomology level.

Combining Theorems (6.7) and (4.11) we obtain the Whitney Duality Theorem for locally flat topological manifolds.

Theorem (6.12). Let $M \subset S$ denote a locally flat imbedding of a topological $n$-manifold $M$ in a topological $(n+k)$-manifold $S$ and let $\left(\overline{\mathscr{T}}_{,} \overline{\mathscr{T}}_{0}\right)$ denote the tangent fiber space of $S$ restricted to $M$. Then

$$
W(M) \smile \bar{W}(M)=W\left(\overline{\mathscr{T}}, \overline{\mathscr{T}}_{0}\right) .
$$

Corollary (6.13). Let $M \subset R^{n+k}$ denote a locally flat imbedding of a topological n-manifold $M$. Then

$$
W(M) \smile \bar{W}(M)=1 .
$$

We now develop the formula of $\mathrm{Wu}(\$ 1)$ and give what is needed to adapt the proof given in Milnor [9]. We will make use of the Poincare Duality for compact topological manifolds and the $X$-product in singular cohomology, both subjects being very carefully presented in Puppe [11]. Let $M$ denote an $n$-manifold, fixed throughout the remainder of this section. Furthermore, let $p_{i}: M \times M \rightarrow M, i=1,2$, denote the usual projections on the first and second factors, respectively, and let $\Delta$ denote the diagonal in $M \times M$. As in Proposition (3.5),

$$
\left(\mathscr{M}, \mathscr{M}_{0}\right)=\left(M \times M, M \times M-\Delta, p_{1}, M\right)
$$

is an $n$-hpb with fiber $(M, M-b), b$ a fixed base point in $M$. If $\left(\mathscr{T}, \mathscr{T}_{0}\right)$ $=\left(T, T_{0}, p, M\right)$ is the tangent fiber space of $M$, there is a natural map (see §3) of fibered pairs, $\psi:\left(\mathscr{T}, \mathscr{T}_{0}\right) \rightarrow\left(\mathscr{M}, \mathscr{M}_{0}\right)$ given by

$$
\psi(\sigma)=(\sigma(0), \sigma(1)), \quad \sigma \in T .
$$

Letting $\left(F, F_{0}\right)$ denote the fiber over $b$ in $\left(\mathscr{T}, \mathscr{T}_{0}\right)$ we recall that

$$
\psi_{0}^{*}: H^{*}(M, M-b ; G) \rightarrow H^{*}\left(F, F_{0} ; G\right)
$$

is an isomorphism for any coefficient group $G$, where $\psi_{0}$ is the restriction of $\psi$ to $\left(F, F_{0}\right)$.

A standard spectral sequence argument then gives the following

Proposition (6.14). If $M$ is G-orientable, the uiove map $\psi$ induces isomorphisms $\psi^{*}: H^{*}(M \times M, M \times M-\Delta ; G) \rightarrow H^{*}\left(T, T_{0} ; G\right)$. 
Let

$$
i:\left(F, F_{0}\right) \rightarrow\left(T, T_{0}\right), j: M \times M \rightarrow(M \times M, M \times M-\Delta), k: M \rightarrow(M, M-b)
$$

denote inclusion maps and $i_{b}:(M, M-b) \rightarrow(M \times M, M \times M-\Delta), f_{b}: M$ $\rightarrow M \times M$ denote sections given by $i_{b}(x)=(b, x), f_{b}(x)=(b, x), x \in M_{0}$. These maps give rise to the following commutative diagram

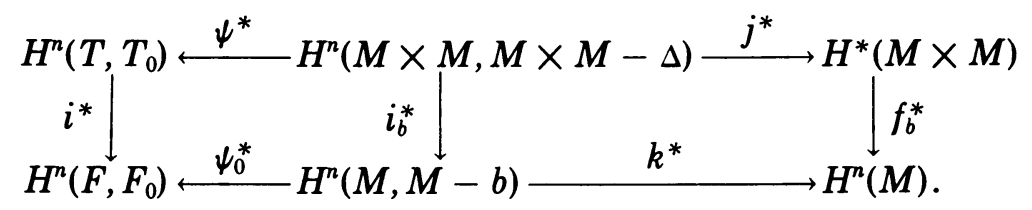

Let $U$ designate the nonzero element of $H^{n}\left(T, T_{0}\right)$ and $U^{\prime}$ that unique element of $H^{n}(M \times M, M \times M-\Delta)$ such that $\psi^{*}\left(U^{\prime}\right)=U$. Furthermore, set $\bar{U}=j^{*}\left(U^{\prime}\right)$ and assume $M$ is compact throughout the remainder of this section.

Proposition (6.15). $f_{b}^{*}(\bar{U})=\mu$, the fundamental class of $H^{n}(M)$.

Proof. Clearly $i_{b}^{*}$ is an isomorphism and since $k^{*}$ is also, we have $k^{*} i_{b}^{*}\left(U^{\prime}\right)$ $=\mu=f_{b}^{*} j^{*}\left(U^{\prime}\right)=f_{b}^{*}(\bar{U})$.

The manifold $M$ is an ANR. Hence, there is a neighborhood $D$ of the diagonal $\Delta$ in $M \times M$ and a map $H: D \times I \rightarrow M$ such that $H(x, y, 0)=x$, $H(x, y, 1)=y, H(x, x, t)=x,(x, y) \in D, 0 \leqq t \leqq 1$. This tells us that if $d: D \rightarrow M \times M$ is the inclusion map that $p_{1} d \sim p_{2} d$. (This $D$ will replace the normal tubular neighborhood of the diagonal which Milnor [9] employs in the differentiable case.)

We now have sufficient material to use the arguments in Milnor [9] and obtain the following

Proposition (6.16). Let $\alpha_{1}, \cdots, \alpha_{n}$ be a basis for $H^{*}(M)$. Then, $\bar{U}$ $=\sum_{i, j} c_{i, j}\left(\alpha_{i} \times \alpha_{j}\right) \in H^{*}(M \times M)$, where the matrix $\left[c_{i j}\right]$ has $\left[y_{k j}\right]$ as its inverse, $y_{k j}=\left\langle\alpha_{k} \cup \alpha_{j}, \bar{\mu}\right\rangle=\left(\alpha_{k} \cup \alpha_{j}\right) \frown \bar{\mu}, \bar{\mu}$ the fundamental class in $H_{n}(M)$.

Now, using the identical argument in $[9$, p. 54] we obtain the $\mathrm{Wu}$ formula. Namely, by Poincaré Duality, there is a unique class $V_{n-i} \in H^{n-i}(M)$ such that

$$
\left\langle S q^{n-i} \alpha, \bar{\mu}\right\rangle=\left\langle V_{n-i} \cup \alpha, \bar{\mu}\right\rangle
$$

for every $\alpha \in H^{i}(M)$. Let $V=V_{0}+V_{1}+\cdots+V_{[n / 2]}$.

THEOREM (6.17) (WU). If $M$ is a compact topological n-manifold and if $W(M)$ is the total Stiefel-Whitney class of $M$ defined in terms of the tangent fiber space of $M$ (Definition (6.3)), then

$$
W(M)=S q V
$$

where $V$ is characterized by the equation 


$$
\langle V \smile \alpha, \bar{\mu}\rangle=\langle S q \alpha, \bar{\mu}\rangle
$$

for all $\alpha \in H^{*}(M)$.

7. The top dual class. Throughout this section $M \subset S$ will represent a locally flat imbedding of the $n$-manifold $M$ in an $(n+k)$-manifold $S$, $\left(\mathscr{T}, \mathscr{T}_{0}\right)$ will denote the tangent fiber space of $M$ and $\left(\mathscr{N}, \mathscr{N}_{0}\right)$ the normal fiber space of this imbedding. Our general objective here is to study the top dual class $\bar{W}_{k}(M)$ associated with this imbedding. The main difficulty in studying this problem in this particular setting is the lack of a normal tubular neighborhood (as far as our present knowledge is concerned) and we need to develop certain techniques which avoid this ignorance.

Let $\left(N, \mathscr{N}_{0}\right)=\left(N, N_{0}, q, M\right)$ designate the normal fiber space above with fiber designated by $\left(F^{\prime}, F_{0}^{\prime}\right)$. Recall that with $\left(\mathscr{T}, \mathscr{T}_{0}\right)$, the tangent fiber space of $M$, there is a useful $n$-hpb $\left(\mathscr{M}, \mathscr{M}_{0}\right)$ (see Proposition (3.5)) associated. In a similar manner, we will associate with $\left(\mathscr{N}, \mathscr{N}_{0}\right)$ a $k$-hpb $\left(\widetilde{T}, \widetilde{\mathscr{N}}_{0}, \tilde{q}, M\right)$, with fiber $\left(\widetilde{F}, \widetilde{F_{0}}\right)$ which will also prove useful. Define

$$
\begin{aligned}
\tilde{N}_{0} & =M \times(S-M), \\
\widetilde{N} & =(M \times(S-M)) \cup \Delta,
\end{aligned}
$$

where $\Delta$ is the diagonal in $M \times M \subset S \times S$. Furthermore, set $\tilde{q}(x, y)=x$, $(x, y) \in \widetilde{N}$. Note that if $b_{0} \in M, \widetilde{F}=\tilde{q}^{-1}\left(b_{0}\right)=(S-M) \cup b_{0}$ and $\widetilde{F_{0}}$ $=\tilde{q}_{0}^{-1}\left(b_{0}\right)=S-M$, where $\tilde{q}_{0}=\tilde{q} \mid \widetilde{N}_{0}$.

Proposition (7.1). $\left(\widetilde{\mathcal{N}}, \widetilde{N_{0}}\right)=\left(\widetilde{N}, \widetilde{N_{0}}, \tilde{q}, \tilde{M}\right)$ is a $k$-hpb with fiber $\left(\widetilde{F}, \widetilde{F_{0}}\right)$.

Proof. The proof that $\left(\widetilde{N}, \widetilde{N}_{0}\right)$ is a locally trivial fibered pair is similar to the corresponding proof for $\left(\mathcal{N}, \mathscr{N}_{0}\right)$, i.e., the proof of Proposition (4.1) and is left to the reader. The homology type of the fiber $\left(\widetilde{F,} \widetilde{F_{0}}\right)$ is seen as follows. Identify the closed $(n+k)$-ball $D^{n+k}$ with a closed neighborhood (in $S$ ) of the base point $b_{0}$ such that $D^{n+k} \cap M=D^{n}$. Let $V=$ interior $D^{n+k}$ and $W=W \cap D^{n}$. Then, by excision, the inclusion map induces isomorphisms

$$
i_{*}: H_{*}\left((V-W) \cup b_{0}, V-W\right) \rightarrow H_{*}\left((S-M) \cup b_{0}, S-M\right),
$$

where $\left((S-M) \cup b_{0}, S-M\right)=\left(\widetilde{F}, \widetilde{F}_{0}\right)$. Just as in Proposition

$$
\left((W-V) \cup b_{0}, W-V\right)=\left(\left(R^{n+k}-R^{n}\right) \cup 0, R^{n+k}-R^{n}\right) \sim\left(R^{k}, R^{k}-0\right)
$$

and hence the homology $H_{*}\left(\tilde{F}, \widetilde{F}_{0}\right)$ is precisely that of $\left(R^{k}, R^{k}-0\right)$.

There is a natural map (of fibered pairs) $\chi:\left(\mathscr{N}, \mathscr{N}_{0}\right) \rightarrow\left(\mathscr{N}, \widetilde{\mathscr{N}_{0}}\right)$, defined as follows

$$
\chi(\omega)=(\omega(0), \omega(1)), \quad \omega \in N .
$$

Let $\chi_{0}:\left(F^{\prime}, F_{0}^{\prime}\right) \rightarrow\left(\widetilde{F}, \widetilde{F}_{0}\right)$ denote the restriction of $\chi$ to $\left(F^{\prime}, F_{0}^{\prime}\right)$. The following commutative diagram 


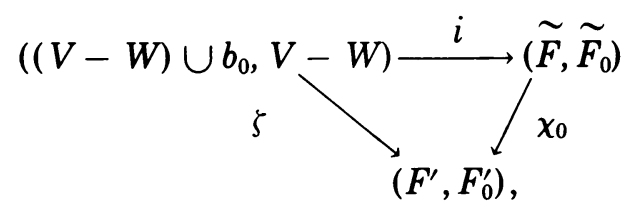

where $\zeta(x)$ is the line segment path (in $V$ ) from $b_{0}$ to $x$, tells us that

$$
\chi_{0 *}: H_{*}\left(F^{\prime}, F_{0}^{\prime} ; G\right) \rightarrow H_{*}\left(\widetilde{F}, \widetilde{F_{0}} ; G\right)
$$

is an isomorphism for any coefficient group $G$, since $\zeta$ is a homotopy equivalence (Proposition (4.1)).

Proposition (7.2). $\left(\widetilde{N}, \widetilde{N}_{0}\right)$ is G-orientable if, and only if, $\left(\mathscr{N}, \mathscr{N}_{0}\right)$ is G-orientable.

Proof. Copy the proof of Proposition (3.13).

Proposition (7.3). If $\left(\mathscr{N}, \mathscr{N}_{0}\right)$ is G-orientable, the above map $x$ induces isomorphisms

$$
\left.\chi_{*}: H_{*}\left(N, N_{0} ; G\right) \rightarrow H_{*}\left(\tilde{\mathscr{N}}, \tilde{\mathscr{N}}_{0}\right) ; G\right) .
$$

Proof. A standard spectral sequence argument as in Proposition (6.14).

Theorem (7.4). Let $X$ denote an ANR (sep. metric), $Y$ a separable metric space and $X \subset Y$, so that $X$ is a closed subset of $Y$. Let $\tilde{N}_{0}=X \times(Y-X)$ and $\tilde{N}=(X \times(Y-X)) \cup \Delta$, where $\Delta$ is the diagonal in $X \times X \subset Y \times Y$. Define $\alpha:\left(\tilde{N}, \tilde{N}_{0}\right) \rightarrow(Y, Y-X)$ by $\alpha(x, y)=y,(x, y) \in \tilde{N}$. Then,

$$
\alpha_{*}: H_{*}\left(\tilde{N}, \widetilde{N}_{0}\right) \rightarrow H_{*}(Y, Y-X)
$$

is an isomorphism (integral coefficients).

Proof. Let $V$ denote an open subset of $Y$ which admits a retraction $r$ : $V \rightarrow X$, onto $X$. By excision, we have an inclusion induced isomorphism

$$
i_{*}: H_{*}(V, V-X) \rightarrow H_{*}(Y, Y-X) .
$$

Let $\widetilde{N}_{0}(V)=X \times(V-X), \widetilde{N}(V)=\widetilde{N}_{0}(V) \cup \Delta$. Again by excision, we have an inclusion induced isomorphism

$$
j_{*}: H_{*}\left(\tilde{N}(V), \tilde{N}_{0}(V)\right) \rightarrow H_{*}\left(\tilde{N}, \tilde{N}_{0}\right) .
$$

Let $\bar{\alpha}$ denote the restriction of $\alpha$ to $\tilde{N}(V)$. Then

$$
\bar{\alpha}:\left(\tilde{N}(V), \tilde{N}_{0}(V)\right) \rightarrow(V, V-X) \text {. }
$$

Define a map

$$
\rho:(V, V-X) \rightarrow\left(\tilde{N}(V), \tilde{N}_{0}(V)\right)
$$

by $\rho(y)=(r(y), y), y \in V$, where $r: V \rightarrow X$ is the previously mentioned retraction. Note that $\bar{\alpha} \rho=1$ and hence $\bar{\alpha}_{*}$ is surjective. Since $\alpha_{*} j_{*}=i_{*} \bar{\alpha}_{*}$, we conclude that $\alpha *$ is surjective. 
Now, let $\Phi=\left\{W_{x}, x \in M\right\}$ denote an open cover of $X$ such that any two maps into $X$ which are $\Phi$-close are homotopic (recall that $X$ is an ANR). For each $x \in M$, choose an open set $U_{x} \subset V$ such that $r\left(U_{x}\right) \subset W_{x}$ and let $U=\bigcup_{x \in M} U_{x} \times U_{x}$. $U$ is then a neighborhood of the diagonal $\Delta$ in $V \times V$. Let $\tilde{N}(U)=\tilde{N} \cap U, \widehat{N}_{0}(U)=\tilde{N} \cap U$. Again excision applies to give us an inclusion induced isomorphism

$$
k_{*}: H_{*}\left(\widetilde{N}(U), \tilde{N}_{0}(U)\right) \rightarrow H_{*}\left(\widetilde{N}, \tilde{N}_{0}\right) .
$$

$\alpha$, restricted to $\tilde{N}(U)$, yields a map

$$
\alpha^{\prime}:\left(\tilde{N}(U), \widetilde{N}_{0}(U)\right) \rightarrow(V, V-X) .
$$

Letting $m:\left(\tilde{N}(U), \tilde{N}_{0}(U)\right) \rightarrow\left(\tilde{N}(V), \tilde{N}_{0}(V)\right)$ denote inclusion map, the following diagram is seen to be homotopy commutative.

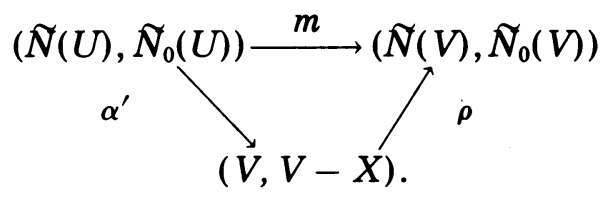

Since $j m=k, m_{*}$ is an isomorphism and hence $\rho_{*} \alpha_{*}^{\prime}$ is an isomorphism. Hence $\alpha_{*}^{\prime}$ is injective. But $\alpha_{*} k_{*}=i_{*} \alpha_{*}^{\prime}$ and hence $\alpha_{*}$ is injective. Thus $\alpha_{*}$ is an isomorphism and our proof is complete.

Theorem (7.5). Suppose the imbedding $M \subset S$ has the additional properties that $M$ is a closed subset of $S$ and the normal fiber space $\left(\mathcal{N}, \mathscr{N}_{0}\right)$ is $G$ orientable. Then, the mapping

$$
\xi:\left(N, N_{0}\right) \rightarrow(S, S-M)
$$

given by $\xi(\omega)=\omega(1)$ induces isomorphisms

$$
\xi_{*}: H_{*}\left(N, N_{0} ; G\right) \rightarrow H_{*}(S, S-M ; G) .
$$

Proof. $\xi=\alpha \chi$ where $\chi *$ is the isomorphism of Proposition (7.4) and $\alpha *$ the isomorphism of Theorem (7.4).

REMARK (7.6). Theorem (7.5), of course, remains valid for cohomology.

The proof of the following proposition follows standard lines and we leave it to the reader.

Proposition (7.7). Suppose $\left(\mathscr{F}, \mathscr{F}_{0}\right),\left(\mathscr{F}^{\prime}, \mathscr{F}_{0}^{\prime}\right)$ are, respectively, $n$ - and $k$-gpb's over the same base $B$ and (Fु", $\left.\mathscr{F}_{0}^{\prime \prime}\right)$ is their Whitney sum. If any two of the three are orientable, so is the third.

Corollary (7.8). If $M \subset S$ is a locally flat imbedding where both $M$ and $S$ are orientable $n$ - and $(n+k)$-manifolds, respectively, then the associated normal fiber space is orientable.

Theorem (7.5) together with the Thom isomorphism gives as an auxiliary 
result the following form of Alexander Duality. Let $\Lambda$ denote a commutative ring with identity. Then, if $\left(\mathscr{N}, \mathscr{N}_{0}\right)$ is $\Lambda$-orientable we have the Thom isomorphism $\phi: H^{i}(M, \Lambda) \rightarrow H^{k+i}\left(N, N_{0} ; \Lambda\right)$ given by $\phi(\alpha)=q^{*}(\alpha) \cup U^{\prime}$, where $U^{\prime}=\phi(1) \in H^{k}\left(N, N_{0} ; \Lambda\right)$. If further $M$ is locally flat in $S$ as a closed subset then the composition $\phi \xi^{*}: H^{k+1}(S, S-M ; \Lambda) \rightarrow H^{i}(M, \Lambda)$ is an isomorphism, where $\xi^{*}$ is the isomorphism of Theorem (7.5) for cohomology.

THEOREM (7.9). If $M \subset S$ is a locally flat imbedding such that the associated normal fiber space is $\Lambda$-orientable (e.g. if both $M$ and $S$ are $\Lambda$-orientable, if $\pi_{1}(M)=0$, or, if $\Lambda=Z_{2}$ ) and if $M$ is a closed subset of $S$, there is an isomorphism

$$
A: H^{i+k}(S, S-M ; \Lambda) \rightarrow H^{i}(M ; \Lambda)
$$

for all $i$, where $k=\operatorname{dim} S-\operatorname{dim} M$. Furthermore, if $\alpha \in H^{k+i}(S, S-M, \Lambda)$, then $A(\alpha)$ is completely characterized by the equation

$$
q^{*}(A(\alpha)) \smile U^{\prime}=\xi^{*}(\alpha) .
$$

Just as in Milnor [9] a $k$-gpb admits an Euler class.

Definition (7.10). The Euler class $X \in H^{k}(B, \Lambda)$ of a $\Lambda$-orientable $k$ gpb over $B$, is given by $\phi^{-1}(U \smile U)$ where $\phi$ is the associated Thom isomorphism and $U=\phi(1)$.

Proposition (7.11). If $\left(\mathscr{F}, \mathscr{F}_{0}\right)=\left(E, E_{0}, \pi, B\right)$ is a $\Lambda$-orientable $k$-gpb and $g: B \rightarrow E$ is any cross-section (at least one exists), then the Euler class $X$ is given by

$$
X=g^{*} i^{*}(U)
$$

where $i: E \rightarrow\left(E, E_{0}\right)$ is inclusion and $U=\phi(1), \phi$ the Thom isomorphism.

Proof. The proof in Milnor [9, Theorem 12] applies with only the following comment necessary. The mapping $\pi: E \rightarrow B$ induces isomorphisms $\pi^{*}: H^{*}(B, \Lambda) \rightarrow H^{*}(E, \Lambda)$ via the Vietoris Mapping Theorem for fiber spaces and singular cohomology. Since $\pi g=1, g^{*} \pi^{*}=1$. Hence, $\pi^{*} g^{*}=1$.

Let us continue now under the assumption that the normal fiber space $\left(\mathcal{N}, \mathscr{N}_{0}\right)$ is $\Lambda$-orientable and set $\bar{W}_{k}(\Lambda)=X \in H^{k}(M ; \Lambda)$ where $X$ is the Euler class of $\left(1,1_{0}\right)$. Also, it is clear that $\bar{W}_{k}\left(Z_{2}\right)=\bar{W}_{k}$ (Definition (6.4)), the top dual class.

Theorem (7.12). Suppose $M \subset S$ is a locally flat imbedding with $M$ closed in Sand $\Lambda$-orientable normal fiber space $\left(\mathcal{N}, \mathscr{N}_{0}\right)$. Suppose $U^{\prime \prime} \in H^{k}(S, S-M ; \Lambda)$ is given by $\xi^{*}\left(U^{\prime \prime}\right)=U^{\prime} \in H^{k}\left(N, N_{0} ; \Lambda\right)$ where $\xi^{*}$ is the cohomology isomorphism of Theorem (7.5). Then,

$$
\bar{W}_{k}(\Lambda)=m^{*} j^{*}\left(U^{\prime \prime}\right),
$$

where $j: S \rightarrow(S, S-M), m: M \rightarrow S$ are inclusions. 
Proof. Let $g: M \rightarrow N$ denote the canonical cross-section, i.e., $g(b)$ is the constant path at $b \in M$. The following diagram is commutative

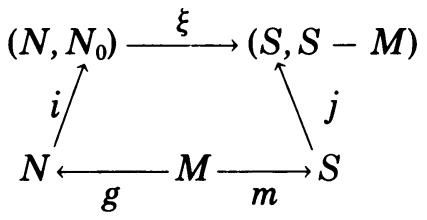

Hence, $m^{*} j^{*}\left(U^{\prime \prime}\right)=g^{*} i^{*} \xi^{*}\left(U^{\prime \prime}\right)=g^{*} i^{*}\left(U^{\prime}\right)=\bar{W}_{k}(\Lambda)$.

Corollary (7.13). Suppose $M \subset R^{n+k}$ is a locally flat imbedding witi $M$ closed in $S$ and $\Lambda$-orientable normal fiber space $\left(\mathcal{N}, \mathscr{N}_{0}\right)$. Then $\bar{W}_{k}(\Lambda)=0$.

REMARK (7.14). The class $U^{\prime \prime} \in H^{k}(S, S-M ; \Lambda)$ is geometrically the following. Choose a point $b \in M$ and let $h: R^{n} \times R^{k} \rightarrow V$ denote a homeomorphism onto a neighborhood $V$ (in $S$ ) of $b$ such that $V \cap M=h\left(R^{n} \times 0\right)$. Then, identifying $R^{k}=0 \times R^{k}, \quad h:\left(R^{k}, R^{k}-0\right) \rightarrow(S, S-M)$ such that $h(0)=b . h$ induces an isomorphism $h^{*}: H^{k}(S, S-M ; \Lambda) \rightarrow H^{k}\left(R^{k}, R^{k}-0 ; \Lambda\right)$ $=\Lambda$ and $h^{*}\left(U^{\prime \prime}\right)=1 \in \Lambda$, the unit element of $\Lambda$. In short, $U^{\prime \prime}$ is determined by a topological $k$-cell which pierces $M$ at one point.

\section{Some geometric applications.}

Definition (8.1). A set $X \subset Y, Y$ a topological space, is said to be instantly deformable into its complement if there exists a homotopy $H: X \times I$ $\rightarrow Y$ such that $H_{0}$ is the inclusion map and $H_{t}(X) \subset Y-X$ for $0<t \leqq 1$.

If $M \subset S$ is a differentiable imbedding, where $M$ and $S$ are differentiable manifolds of dimensions $n$ and $n+k$, respectively, then $M$ is instantly deformable into its complement when $k>n$. This is because the classical normal sphere bundle is a $(k-1)$-sphere bundle and all obstructions to finding a cross-section vanish. In fact, if the imbedding is such that the associated normal bundle is orientable, then this result remains valid for $k \geqq n$, provided $S=R^{n+k}$. We now proceed to investigate the corresponding result for locally flat imbeddings of topological manifolds.

Lemma (8.2). Suppose $(E, p, B)$ is a fiber space and $g: B^{\prime} \rightarrow B$ is a dominating map, i.e., there is also a map $h: B \rightarrow B^{\prime}$ such that $g h \sim 1$. Then, if $\left(E^{\prime}, p^{\prime}, B^{\prime}\right)$ is the fiber space induced by $g, p$ admits a cross-section if, and only if, $p^{\prime}$ does.

Proof. Recall that $E^{\prime}=\left\{\left(b^{\prime}, e\right) \in B^{\prime} \times E \mid g\left(b^{\prime}\right)=p(e)\right\}$. Let $p^{\prime}\left(b^{\prime}, e\right)=b^{\prime}$ and $g^{\prime}\left(b^{\prime}, e\right)=e$ and we have the usual commutative diagram

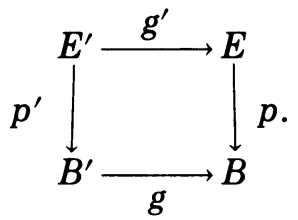


If $p$ admits a cross-section $\sigma$, then $\sigma^{\prime}$, given by $\sigma^{\prime}\left(b^{\prime}\right)=\left(b^{\prime}, \sigma g(b)\right)$ provides a cross-section for $p^{\prime}$. On the other hand, if $\sigma^{\prime}$ is a cross-section for $p^{\prime}$, set $\tau(b)=g^{\prime} \sigma^{\prime} h(b)$, where $h$ is a right homotopy inverse for $g$. Now, $p \tau$ $=p q^{\prime} \sigma^{\prime} h=g p^{\prime} \sigma^{\prime} h=g h \sim 1$. Thus, $p \tau \sim 1$. This is sufficient (see p. 15 of [5]) to insure the existence of a cross-section $\sigma$ for $p$.

Theorem (8.3). Suppose $M \subset S$ is a locally flat imbedding of a topological $n$-manifold $M$ in a topological $(n+k)$-manifold $S(M$ need not be closed in $S)$. Then, if $k>n, M$ is instantly deformable into its complement.

Proof. Let $\left(\mathcal{N}, \mathscr{N}_{0}\right)=\left(N, N_{0}, q, M\right)$ denote the associated normal fiber space which is a $k$-gpb. Then, $\left(N_{0}, q_{0}, M\right)$ is a fiber space with fiber $F_{0} \sim R^{k}$ $-0 \sim S^{k-1}$, a $(k-1)$-sphere, where $(k-1) \geqq n$. Since $M$ is an ANR (sep. metric) and also of dimension $n$, there is an $n$-polyhedron $P$ (locally finite) and a dominating map $g: P \rightarrow M$. Let $\left(N_{0}^{\prime}, q_{0}^{\prime}, P\right)$ denote the fiber space over $P$ induced by the map $g$. A standard obstruction argument applied to $\left(N_{0}^{\prime}, q_{0}^{\prime}, P\right)$ yields a cross-section for $q_{0}^{\prime}$ and hence by Lemma (8.2), there is a cross-section $\sigma$ for $q_{0}$. But $N_{0}$ consists precisely of paths which start at a point of $M$ and never hit $M$ again. In particular, if $b \in M, \sigma(b)(0)=b$, and $\sigma(b)(t) \in S-M$ for $0<t \leqq 1$. The required deformation $H: M \times I \rightarrow S$ is then given by

$$
H(b, t)=\sigma(b)(t), \quad 0 \leqq t \leqq 1 .
$$

REMaRK (8.4). Theorem (8.3) for the case $k=n$ remains valid if $M \subset R^{n+k}$ in a locally flat manner and if furthermore $M$ is closed in $R^{n+k}$ and the associated normal fiber space is orientable. The proof of this fact requires a development of more obstruction theory in this setting and this will be discussed in another work.

Suppose $M \subset R^{n+k}$ and one has given a tubular neighborhood $\left(U, U_{0}, p, M\right)$ (Definition (4.4) of $M$ ). This tubular neighborhood may be given by topological methods and not through the normal bundle of a differentiable imbedding. The question as to whether this tubular neighborhood is actually a product is of interest.

Theorem (8.5). Let $M \subset R^{n+k}$, where $M$ is a topological n-manifold, and $\left(U, U_{0}, p, M\right)$ a tubular neighborhood of $M$ in $R^{n+k}$. Then, if $\left(U, U_{0}, p, M\right)$ is fiber homotopically trivial, $W(M)=1$.

Proof. First observe that the imbedding $M \subset R^{n+k}$ is necessarily locally flat. Hence, we may consider $\left(\mathcal{N}, \mathscr{N}_{0}\right)$ the associated normal fiber space. By Proposition (4.8), $\left(U, U_{0}, p, M\right) \sim_{f}\left(\mathcal{N}, \mathscr{N}_{0}\right)$ and hence if $\left(U, U_{0}, p, M\right)$ is fiber homotopically trivial, so is $\left(\mathcal{N}, \mathscr{N}_{0}\right)$. This would imply $\bar{W}(M)=1$ and since $W(M) \smile \bar{W}(M)=1$ we have, necessarily, $W(M)=1$.

Added in proof. If $M$ is a topological $n$-manifold, the group $G(M)$ of 
self-homeomorphisms of $M$ is a topological group, using the c-o topology, without the assumption of compactness. The proof of this is simple and direct. Alternatively, a more general result of William Browder says that if $X$ is locally compact and locally connected, $G(X)$ is a topological group in the c-o topology. These remarks are pertinent to Remarks (3.6), (3.11), (4.2) and to slightly simplify a few arguments in $\$ 3$ and 4 . Finally, the "conjecture" in Remark (4.6) is not difficult to establish and the author is indebted to James Kister for this observation.

\section{BiBLIOGRAPHY}

1. G. Allaud and E. Fadell, A fiber homotopy extension theorem, Trans. Amer. Math. Soc. 104 (1962), 239-251.

2. A. Dold, Partitions of unity in the theory of fibrations, Ann. of Math. (2) 78 (1963), 223-255.

3. E. Fadell, On fiber spaces, Trans. Amer. Math. Soc. 90 (1959), 1-14.

4.

5. E. Fadell and L. Neuwirth, Configuration spaces, Math. Scand. 10 (1962), 111-118.

6. A. Haefliger, Points multiples d'une application et produit cyclique réduit, Amer. J. Math. 83 (1961), 57-70.

7. S. T. Hu, Fibrings of enveloping spaces, Proc. London Math. Soc. (3) 11 (1961), 691-707.

8. W. Hurewicz, On the concept of fibre space, Proc. Nat. Acad. Sci. U.S.A. 41 (1955), 956-961.

9. J. Milnor, Lectures on characteristic classes (Mimeographed Notes), Princeton, 1957.

10. J. Nash, A path space and the Stiefel-Whitney classes, Proc. Nat. Acad. Sci. U.S.A. 41 (1955), 320-321.

11. D. Puppe, Topologie. II, Universität Bonn, 1960.

12. J.-P. Serre, Homologie singuliere des espaces fibrés, Ann. of Math. (2) 54 (1951), 425-505.

13. W. T. Wu, Classes charactéristiques et i-carrés d'une variété, C. R. Acad. Sci. Paris 230 (1950), 508-511.

UNIVERSITY OF WISCONSIN, Madison, Wisconsin 